TITLE:

\title{
Multidimensional vibrational spectroscopy for tunneling processes in a dissipative environment
}

\author{
$\operatorname{AUTHOR}(\mathrm{S}):$
}

Ishizaki, A; Tanimura, Y

\section{CITATION:}

Ishizaki, A ...[et al]. Multidimensional vibrational spectroscopy for tunneling processes in a dissipative environment. The Journal of Chemical Physics 2005, 123(1): 014503.

\section{ISSUE DATE:}

2005-07-01

URL:

http://hdl.handle.net/2433/50559

\section{RIGHT:}

Copyright 2005 American Institute of Physics. This article may be downloaded for personal use only. Any other use requires prior permission of the author and the American Institute of Physics. 


\title{
Multidimensional vibrational spectroscopy for tunneling processes in a dissipative environment
}

\author{
Akihito Ishizaki $^{\mathrm{a})}$ \\ Department of Physics, Graduate School of Science, Kyoto University, Kyoto 606-8502, Japan \\ Yoshitaka Tanimurab) \\ Department of Chemistry, Graduate School of Science, Kyoto University, Kyoto 606-8502, Japan
}

(Received 21 January 2005; accepted 18 March 2005; published online 13 July 2005)

\begin{abstract}
Simulating tunneling processes as well as their observation are challenging problems for many areas. In this study, we consider a double-well potential system coupled to a heat bath with a linear-linear (LL) and square-linear (SL) system-bath interactions. The LL interaction leads to longitudinal $\left(T_{1}\right)$ and transversal $\left(T_{2}\right)$ homogeneous relaxations, whereas the SL interaction leads to the inhomogeneous dephasing $\left(T_{2}{ }^{*}\right)$ relaxation in the white noise limit with a rotating wave approximation. We discuss the dynamics of the double-well system under infrared (IR) laser excitations from a Gaussian-Markovian quantum Fokker-Planck equation approach, which was developed by generalizing Kubo's stochastic Liouville equation. Analytical expression of the Green function is obtained for a case of two-state-jump modulation by performing the Fourier-Laplace transformation. We then calculate a two-dimensional infrared signal, which is defined by the four-body correlation function of optical dipole, for various noise correlation time, system-bath coupling parameters, and temperatures. It is shown that the bath-induced vibrational excitation and relaxation dynamics between the tunneling splitting levels can be detected as the isolated off-diagonal peaks in the third-order two-dimensional infrared (2D-IR) spectroscopy for a specific phase matching condition. Furthermore, this spectroscopy also allows us to directly evaluate the rate constants for tunneling reactions, which relates to the coherence between the splitting levels; it can be regarded as a novel technique for measuring chemical reaction rates. We depict the change of reaction rates as a function of system-bath coupling strength and a temperature through the 2D-IR signal. () 2005 American Institute of Physics. [DOI: 10.1063/1.1906215]
\end{abstract}

\section{INTRODUCTION}

Tunneling process in a dissipative environment has enormous importance for many areas in physics, chemistry, and biology. ${ }^{1-3}$ For the purpose of understanding the process, numerous theoretical studies have been devoted from analytical and computational approaches compared with experimental results. Nevertheless, many interesting problems are yet left for study due to the difficulties of theoretical treatments and experimental observations.

In this paper, we employed a Gaussian-Markovian quantum Fokker-Planck equation to study dissipative tunneling dynamics including a nonlinear system-bath interaction, which had not been taken into account in former studies. Our main purpose is, however, not only to develop a theoretical method, but also to demonstrate the advantage of analyzing the tunneling process by means of two-dimensional spectroscopy. Traditionally the tunneling process has been characterized by a chemical reaction rate $^{4-8}$ Unfortunately, the change of chemical reaction rate as a function of temperature or system-bath coupling does not easily reveal microscopic details of the underlying mechanism and process involved in tunneling. In a gas phase, absorption, ${ }^{9,10}$ electronically

\footnotetext{
${ }^{a)}$ Electronic mail: ishizaki@kuchem.kyoto-u.ac.jp

${ }^{b}$ Electronic mail: tanimura@ @uchem.kyoto-u.ac.jp
}

resonant ${ }^{11}$ or microwave spectroscopy ${ }^{12}$ may provide important information such as the frequency of the tunneling splitting levels; however, in a condensed phase, one cannot clearly extract transition rates between different levels because the spectra are usually broadened and featureless due to the environment in which much information is buried.

Both the reaction rate and infrared (IR) absorption spectrum are characterized by two-body correlation functions of physical operators as $\langle[\hat{\mathcal{O}}(t), \hat{\mathcal{O}}]\rangle$, where $\hat{\mathcal{O}}$ is the probability density or flux for reaction rate, ${ }^{4-6}$ and the dipole operator for IR absorption spectrum. ${ }^{13-15}$ It is generally the case for one-dimensional observables-those that are characterized by two-body correlation functions, and hence have one independent time or frequency variable-that one cannot uniquely extract information on the molecular system in the condensed phase. To solve this problem, one needs to explore multidimensional observables which can be characterized by multibody correlation functions, even though one has to overcome many theoretical and experimental difficulties.

Among many tunneling processes, here we focus on a proton transfer (PT) reaction process, which has been a subject of the great interest in spectroscopy. We then explore multidimensional vibrational spectrum, which is the optical analog of the multidimensional NMR techniques and has recently been investigated by many groups. ${ }^{16,17}$ The distinctive advantage of the multidimensional vibrational spectroscopy 
is due to the multiple pumping and probing processes, which can be described by the multibody correlation functions of the polarizability or the dipole moment as a function of the relevant vibrational coordinate. For any physical operator $\hat{\mathcal{O}}$ the $n$-body correlation function is expressed as

$$
\begin{aligned}
C_{\mathcal{O}}^{(n)} & \left(t_{n-1}, t_{n-2}, \ldots, t_{1}\right) \\
& =\left\langle\left[\left[\left[\left[\hat{\mathcal{O}}\left(t_{n-1}\right), \hat{\mathcal{O}}\left(t_{n-2}\right)\right], \ldots\right], \hat{\mathcal{O}}\left(t_{1}\right)\right], \hat{\mathcal{O}}\right]\right\rangle,
\end{aligned}
$$

where $\hat{\mathcal{O}}(t)$ is the Heisenberg operator of $\hat{\mathcal{O}}$ and $\langle\cdots\rangle$ means the ensemble average. For Raman and IR case, $\hat{\mathcal{O}}$ corresponds to the polarizability and the dipole moment, respectively; the third-order Raman and second-order IR processes are represented by its three-body correlation function, whereas the seventh-order Raman and third-order IR are by its four-body correlation function. ${ }^{15,16}$ If the system is harmonic and the polarizability or the dipole moment is the linear function of molecular coordinate, then the three-body correlation function will be vanished due to the Gaussian integrals involved in the thermal average. The four-body correlation function will be also vanished due to the destructive contribution of the coherence involved in the different Liouville paths of optical process. Thus the presence of the nonlinear coordinate dependence of the polarizability or dipole and the anharmonicity of potential or system-bath interaction, etc., is essential to have a signal. In linear spectroscopy which is defined by a two-body correlation function, the main contribution of the signal arises from harmonic vibrational motion; nonharmonic effects are merely the small correction of the harmonic contribution; however, in higherorder vibrational spectroscopy, anharmonicity leads the leading order contribution. Therefore, one may consider that linear vibrational spectroscopy is the spectroscopy to see the character of harmonic vibrational motions, whereas the higher-order vibrational spectroscopy is the spectroscopy to detect nonharmonic kinetics.

Consequently, multidimensional spectroscopy has been proven to be a valuable and versatile tool for diverse topics in the condensed phases as the anharmonisity of potentials, ${ }^{18-20}$ vibrational mode coupling, ${ }^{21-26}$ vibrational dephasing mechanisms, ${ }^{27-32}$ bath-induced coherence transfer processes, ${ }^{33}$ and structural changes of large molecules. ${ }^{17,34-39}$ For fifth-order Raman spectroscopy experiments, signals corresponding to various Raman polarizability tensor elements were measured for intermolecular vibrational modes of liquid $\mathrm{CS}_{2}$ (Refs. 40-42) and solutions of $\mathrm{CS}_{2}$ (Ref. 43) by minimizing the cascade contributions, ${ }^{44,45}$ which were underestimated in the initial attempts of experiments. ${ }^{46-48}$ For the third-order IR experiments, the femtosecond phasecontrolled IR pulses are now available to obtain the heterodyne detected signals from the matter. ${ }^{36}$ The twodimensional plots of the three-pulse vibrational echo technique were applied to the conformational fluctuation of an $\alpha$-helical peptide, ${ }^{49,50}$ model dipeptides, ${ }^{51,52}$ molecular stretching mode, ${ }^{53}$ and hydrogen bonding interaction between solute and solvent. ${ }^{54-58}$

In this paper, we examine this sensitivity to study a dissipative tunneling dynamics. To investigate, we employ one- dimensional double-well potential system. The simplicity of the model system, where the reaction coordinate is restricted to one dimension, may limit the value of any direct comparisons to actual future experiments. However, the use of a simple system is advantageous in clarifying the issues that must be considered in realistic and experimental cases. Since we used energy eigenstates representation instead of coordinate states, the extension to multidimensional potential system is straight forward, although the number of the eigenstates to be included increased dramatically as the dimension of coordinate increases, which makes numerical calculation very difficult.

To include dissipation, one commonly employs different models and assumptions for an optical case and a chemical reaction case. In optics, PT system has been studied by a discrete energy eigenstate system coupled with a white noise heatbath in rotating wave approximation (RWA) form; the dynamics of the system is described by the longitudinal $\left(T_{1}\right)$ and transverse $\left(T_{2}\right)$ relaxation times. In addition, the effect of vibrational dephasing is incorporated by stochastic frequency modulation which is characterized by $T_{2}{ }^{*}$ in the motional narrowing limit. In such limit, the equation of motion reduces to the optical Bloch equation or Redfield equation, which are characterized by the simple relaxation constants $\kappa_{1}=1 / T_{1}$ and $\kappa_{2}=1 / 2 T_{2}+1 / T_{2}{ }^{*}$. On the other hand, in the study of chemical reaction process, the system is expressed in molecular coordinates linearly coupled (LL interaction) to the heatbath (Brownian model). The dynamics of the system is studied by path-integral approach ${ }^{59}$ or reduced equation of motion approach ${ }^{8,60-62}$ without RWA for colored noise. The framework of the theory is therefore more general than the energy eigenstate model; however, the LL interaction leads to only $T_{1}$ and $T_{2}$ relaxation in the white noise case. In order to include the vibrational dephasing, one therefore includes a square-linear (SL) coupling ${ }^{27,28,63}$ in addition to the LL interaction. The SL interaction reduces not only vibrational dephasing but also energy transfer. What is more important about this interaction is the cross-term contribution between $\mathrm{LL}+\mathrm{SL},{ }^{29,30}$ which gives rise to such important effect as the coherent transfer. ${ }^{33,64}$ We solve this model for colored noise without RWA at finite temperature.

In this paper, we investigate the nonadiabatic PT reaction system, where the reaction barrier is rather high compared with the thermal activation energy and consequently the reaction occurs via tunneling, in the condensed phase. Specifically, we demonstrate the ability of two-dimensional IR spectroscopy to separate and observe directly the vibrational excitation and relaxation dynamics of interest; this allows us to directly evaluate the rate constants for tunneling chemical reactions in the dissipative environment.

The paper is organized as follows: In Sec. II we introduce the model for a nonadiabatic PT system in the condensed phase. In Sec. III we derive the third-order IR response function by the Gaussian-Markovian quantum Fokker-Planck equation approach. In Sec. IV numerical results are presented for the two-dimensional (2D) IR signal and are discussed. Here, it is shown that the bath-induced vibrational excitation and relaxation dynamics between the tunneling splitting levels can be detected as the isolated off- 


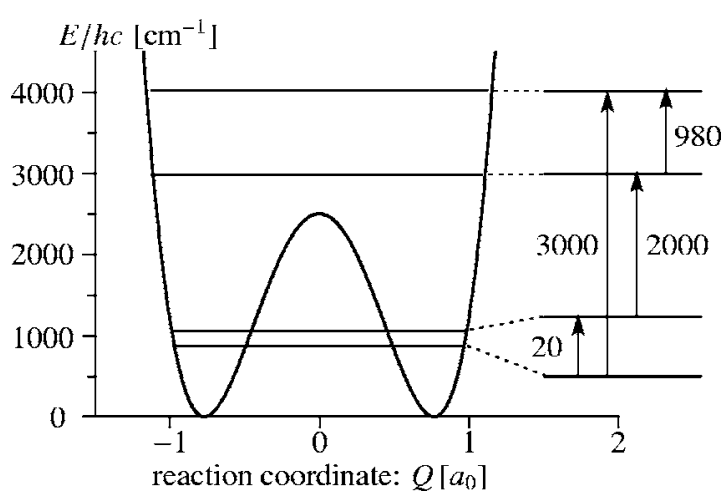

FIG. 1. Potential Eq. (2) with the lowest eigenstates for $Q_{0}=0.76 a_{0}$ and $U_{0}=2500 \mathrm{~cm}^{-1}$. These parameters give rise to a tunneling splitting frequency $\omega_{10}=20 \mathrm{~cm}^{-1}$. The dipole-allowed IR transitions are shown as well; we set $\omega_{30}=3000 \mathrm{~cm}^{-1}$ and $\omega_{21}=2000 \mathrm{~cm}^{-1}$ for clarity. In this study, the four lowest states have been taken into account.

diagonal peaks in the third-order 2D-IR spectroscopy for a specific phase matching condition. Furthermore, it is also demonstrated that this spectroscopy also allows us to directly evaluate the rate constants for tunneling chemical reactions, which relates to the coherence between the splitting levels. Finally, Sec. V is devoted to conclusion remarks.

\section{THE DISSIPATIVE PROTON TRANSFER SYSTEM}

The PT dynamics in principle is a multidimensional process. ${ }^{64}$ We will, however, treat the PT dynamics as a onedimensional process in order to keep our model as simple as possible such that the information content of twodimensional IR spectroscopy can be clearly identified. We consider the one-dimensional double-well system $A$ defined by the Hamiltonian,

$$
\hat{H}_{A}=\frac{\hat{P}^{2}}{2 M}+\frac{U_{0}}{Q_{0}{ }^{4}}\left(\hat{Q}-Q_{0}\right)^{2}\left(\hat{Q}+Q_{0}\right)^{2},
$$

where $M, \hat{Q}$, and $\hat{P}$ denote the mass of a proton, the reaction coordinate, and the conjugate momentum to $\hat{Q}$, respectively, and $U_{0}$ is the height of the reaction barrier separating the two minima which are located at $\pm Q_{0}$ as shown in Fig. 1. All other degrees of freedom of the total system, i.e., intramolecular and solvent modes, are comprised in bath modes. In the energy eigenstates representation, the Hamiltonian of the system $A$ is expressed as $\hat{H}_{A}|j\rangle=E_{j}|j\rangle$. The tunneling processes are characterized by the pairs of states separated by tunneling splitting energy. We introduce a transition frequency between two states $\hbar \omega_{j k} \equiv E_{j}-E_{k}$. From the instanton approach, ${ }^{65,66}$ the tunneling splitting frequency in the vibrational ground state for the system $A$ is obtained as

$$
\omega_{10}=\omega_{\text {well }} \sqrt{\frac{128 U_{0}}{\pi \hbar \omega_{\text {well }}}} \exp \left(-\frac{16 U_{0}}{3 \hbar \omega_{\text {well }}}\right),
$$

where $\omega_{\text {well }}\left(\sim \omega_{21}\right)$ is the characteristic dynamical frequency of the system:

$$
\omega_{\mathrm{well}}=\sqrt{\frac{8 U_{0}}{M Q_{0}^{2}}} .
$$

The nonadiabatic or deep tunneling limit of the PT reaction is characterized by the following condition: ${ }^{67}$

$$
\hbar \omega_{10} \ll k_{B} T \ll \hbar \omega_{\text {well }},
$$

where $k_{B}$ and $T$ are the Boltzmann constant and the temperature, respectively.

The Hamiltonian of the total system is assumed to be of the form

$$
\hat{H}_{\mathrm{tot}}=\hat{H}_{A}+\sum_{j}\left[\frac{\hat{p}_{j}^{2}}{2 m_{j}}+\frac{1}{2} m_{j} \omega_{j}^{2}\left(\hat{x}_{j}-\frac{c_{j} V(\hat{Q})}{m_{j} \omega_{j}^{2}}\right)^{2}\right],
$$

where the bath degrees of freedom are treated as an ensemble of harmonic oscillators, and the coordinate, momentum, mass, and frequency of the $j$ th bath oscillator are given by $\hat{x}_{j}, \hat{p}_{j}, m_{j}$, and $\omega_{j}$, respectively.

In Eq. (6), the system-bath interaction is expressed as

$$
\hat{H}_{\mathrm{I}}=-\sum_{j} c_{j} \hat{x}_{j} V(\hat{Q})
$$

where $c_{j}$ denotes the coupling strength to the $j$ th bath mode. For the bath coordinate $\hat{x}_{j}$ we take into account only the linear dependence; for the system coordinate $\hat{Q}$ we include terms up to second order,

$$
V(\hat{Q})=K_{\mathrm{LL}} \hat{Q}+\frac{K_{\mathrm{SL}}}{2} \hat{Q}^{2} .
$$

Constants $K_{\mathrm{LL}} \geqslant 0$ and $K_{\mathrm{SL}}$ are introduced to specify the relative importance of the couplings via the $\hat{Q}$ and $\hat{Q}^{2}$ terms, respectively. We refer to the term proportional to $K_{\mathrm{LL}}$ as the LL coupling term, and the term proportional to $K_{\mathrm{SL}}$ as the SL coupling term. Conventionally, Brownian oscillator models with only the LL coupling term have been used to explore the dissipative effects on quantum processes in the condensed phase. In spectroscopy, the LL term is responsible for energy dissipation from the vibrational system to the environment, which is referred as the population decay and dephasing in the white noise limit with RWA, i.e., the $T_{1}$ and $T_{2}$ processes in NMR. This term is, however, insufficient to induce vibrational pure dephasing due to level fluctuations or the $T_{2}{ }^{*}$ process in the white noise limit. In order to introduce the pure dephasing, we have to incorporate the SL coupling term $c_{j} \hat{x}_{j} \cdot K_{\mathrm{SL}} \hat{Q}^{2} / 2 .^{27,28,63}$ This term induces frequency modulations of the relevant system. For instance, for a harmonic oscillator system with a fundamental frequency $\omega_{0}$, the instantaneous frequency modulations is expressed as

$$
\omega(t)=\sqrt{\omega_{0}^{2}+\sum_{j} c_{j} K_{\mathrm{SL}} x_{j}(t) / M} .
$$

Furthermore, in addition to the LL interaction, this interaction reduces not only vibrational dephasing but also energy transfer. What is more important about this interaction is the cross-term contribution between $\mathrm{LL}+\mathrm{SL},{ }^{29,30}$ which gives rise to important effect for multidimensional spectroscopy. For the PT system, it is known that such mode promotes the 
transfer event by effectively reducing the reaction barrier. ${ }^{64}$

The bath dynamics-and hence the system-bath correlation - can be characterized by the spectral distribution function defined by

$$
J(\omega)=\sum_{j=1}^{N} \frac{c_{j}^{2}}{2 m_{j} \omega_{j}} \delta\left(\omega-\omega_{j}\right) .
$$

We should notice that the typical relaxation time for the bath cannot be negligibly small when we discuss ultrafast phenomena such as proton transfer dynamics. Instead of Gaussian-white noise, we must therefore consider a colored noise bath; namely, a Gaussian-Markovian noise bath. The spectral distribution function, Eq. (10), for the GaussianMarkovian noise bath is given by the Ohmic form with the Lorentzian cutoff: ${ }^{68}$

$$
J^{\mathrm{GM}}(\omega)=\frac{M \zeta}{\pi} \frac{\gamma^{2} \omega}{\gamma^{2}+\omega^{2}} .
$$

Here, the constant $\gamma$ represents the width of the spectral distribution of the bath modes and is related to the correlation time of the noise induced by the bath:

$$
\tau_{c}=1 / \gamma .
$$

To see this relationship, we define the collective bath coordinate of the bath modes as

$$
\hat{X}=\sum_{j} c_{j} \hat{x}_{j} .
$$

Within the high-temperature approximation $\beta \hbar \gamma \ll 1(\beta$ $\left.=1 / k_{\mathrm{B}} T\right)$, the symmetrized correlation function of the collective bath coordinate takes a single exponential decay form ${ }^{68}$

$$
\frac{1}{2}\langle\hat{X}(t) \hat{X}(0)+\hat{X}(0) \hat{X}(t)\rangle_{B}=\frac{M \zeta \gamma}{\beta} e^{-\gamma|t|},
$$

where $\hat{X}(t)$ is the Heisenberg representation of $\hat{X}$ and $\langle\cdots\rangle_{B}$ means taking the thermal average with respect to the bath degrees of freedom. Equation (14) states that the bath oscillators disturb the system $A$ with Gaussian-Markovian noise. $\zeta$ is related to the system-bath coupling strength. The LL coupling strength and the SL coupling strength are defined from Eqs. (8) and (11) by

$$
\zeta_{\mathrm{LL}}=K_{\mathrm{LL}} \zeta
$$

and

$$
\zeta_{\mathrm{SL}}=\frac{K_{\mathrm{SL}}^{2}}{4} \zeta,
$$

respectively. Therefore, a set of four parameters $\zeta_{\mathrm{LL}}, \zeta_{\mathrm{SL}}$, $\tau_{c}=1 / \gamma$, and $\beta$ completely specified the system-bath coupling of our model.

\section{IR RESPONSE FUNCTION}

The response of the molecular system to an external laser field $E(\mathbf{r}, t)$ is conveniently described in terms of multibody correlation functions. In the third order with respect to $E(\mathbf{r}, t)$, the relevant macroscopic polarization is formally given by ${ }^{15}$
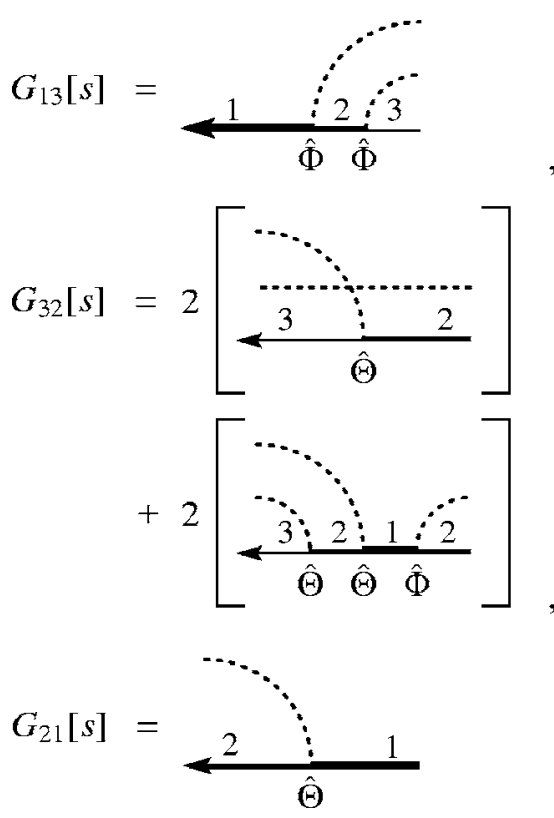

FIG. 2. Examples of the diagrammatic expression for $G_{J K}[s]$. A broken line denotes the one-photon excitation associated with $\hat{\Theta}$ and $\hat{\Phi}$.

$$
\begin{aligned}
P^{(3)}(\mathbf{r}, t)= & \int_{0}^{\infty} d t_{3} \int_{0}^{\infty} d t_{2} \int_{0}^{\infty} d t_{1} R_{\mathrm{IR}}^{(3)}\left(t_{3}, t_{2}, t_{1}\right) E\left(\mathbf{r}, t-t_{3}\right) \\
& \times E\left(\mathbf{r}, t-t_{3}-t_{2}\right) E\left(\mathbf{r}, t-t_{3}-t_{2}-t_{1}\right),
\end{aligned}
$$

with the third-order IR response function

$$
\begin{aligned}
R_{\mathrm{IR}}^{(3)}\left(t_{3}, t_{2}, t_{1}\right)= & \left(\frac{i}{\hbar}\right)^{3} \operatorname{Tr}\left\{\left[\left[\left[\hat{\mu}\left(t_{3}+t_{2}+t_{1}\right),\right.\right.\right.\right. \\
& \left.\left.\left.\left.\hat{\mu}\left(t_{2}+t_{1}\right)\right], \hat{\mu}\left(t_{1}\right)\right], \hat{\mu}(0)\right] \hat{\rho}_{\mathrm{tot}}^{\mathrm{eq}}\right\},
\end{aligned}
$$

where $\hat{\mu}(t)=e^{i \hat{H}_{\mathrm{tot}^{\mathrm{t}}} / \hbar} \mu(\hat{Q}) e^{-i \hat{H}_{\mathrm{tot}} t / \hbar}$ is the Heisenberg representation of the dipole operator and $\hat{\rho}_{\mathrm{tot}}^{\mathrm{eq}}=e^{-\beta \hat{H}_{\mathrm{tot}}} / \operatorname{Tr}\left\{e^{-\beta \hat{H}_{\mathrm{tot}}}\right\}$ is the thermal equilibrium density operator of the total system. In order to simplify expressions here and hereafter, we introduce the notation, ${ }^{69}$

$$
\hat{\mathcal{O}}^{\times} \hat{f} \equiv[\hat{\mathcal{O}}, \hat{f}],
$$

for a hyperorator $\hat{\mathcal{O}}^{\times}$which constructs a commutator $[\hat{\mathcal{O}}, \hat{f}]$ with an operand operator $\hat{f}$. Using this notation, we can recast the IR response function given by Eq. (18) into

$$
\begin{aligned}
R_{\mathrm{IR}}^{(3)}\left(t_{3}, t_{2}, t_{1}\right)= & \operatorname{Tr}\left\{\mu(\hat{Q}) e^{-i \hat{\mathcal{L}}_{\mathrm{tot}^{t}} \frac{i}{\hbar}} \mu(\hat{Q})^{\times} e^{-i \hat{\mathcal{L}}_{\mathrm{tot}} t_{2}} \frac{i}{\hbar}\right. \\
& \left.\times \mu(\hat{Q})^{\times} e^{-i \hat{\mathcal{L}}_{\mathrm{tot}^{t}} \frac{i}{\hbar}} \mu(\hat{Q})^{\times} \hat{\rho}_{\mathrm{tot}}^{\mathrm{eq}}\right\},
\end{aligned}
$$

where $i \hat{\mathcal{L}}_{\text {tot }} \equiv(i / \hbar) \hat{H}_{\text {tot }} \times$ is the Liouvillian of the total system.

Here let us introduce the three-dimensional Laplace transform of $R_{\mathrm{IR}}^{(3)}\left(t_{3}, t_{2}, t_{1}\right)$ as

$$
\begin{aligned}
R_{\mathrm{IR}}^{(3)}\left[s_{3}, s_{2}, s_{1}\right]= & \int_{0}^{\infty} d t_{3} \int_{0}^{\infty} d t_{2} \int_{0}^{\infty} d t_{1} e^{-s_{3} t_{3}-s_{2} t_{2}-s_{1} t_{1}} \\
& \times R_{\mathrm{IR}}^{(3)}\left(t_{3}, t_{2}, t_{1}\right),
\end{aligned}
$$

and then Eq. (20) becomes 


$$
\begin{aligned}
R_{\mathrm{IR}}^{(3)}\left[s_{3}, s_{2}, s_{1}\right]= & \operatorname{Tr}\left\{\mu(\hat{Q}) \frac{1}{s_{3}+i \hat{\mathcal{L}}_{\text {tot }}} \frac{i}{\hbar} \mu(\hat{Q})^{\times} \frac{1}{s_{2}+i \hat{\mathcal{L}}_{\text {tot }}}\right. \\
& \left.\times \frac{i}{\hbar} \mu(\hat{Q})^{\times} \frac{1}{s_{1}+i \hat{\mathcal{L}}_{\mathrm{tot}}} \frac{i}{\hbar} \mu(\hat{Q})^{\times} \hat{\rho}_{\mathrm{tot}}^{\mathrm{eq}}\right\},
\end{aligned}
$$

where the fractional operators indicate the corresponding inverse operators.

\section{A. In a case of Gaussian-Markovian bath}

Because the laser field is assumed to only interact with the system $A$ via the dipole $\mu(Q)$, the reduced description of the optical processes can be made. By tracing over the optically inactive bath degrees of freedom from Eq. (22), we obtain the reduced response function of the relevant system as (see Fig. 3 and, Appendixes A and B)

$$
R_{\mathrm{IR}}^{(3)}\left[s_{3}, s_{2}, s_{1}\right]=\sum_{J=1}^{\infty} \sum_{K=1}^{\infty} \sum_{L=1}^{\infty} \operatorname{Tr}_{A}\left\{\mu(\hat{Q}) \hat{G}_{1 J}\left[s_{3}\right] \frac{i}{\hbar} \mu(\hat{Q})^{\times} \hat{G}_{J K}\left[s_{2}\right] \frac{i}{\hbar} \mu(\hat{Q})^{\times} \hat{G}_{K L}\left[s_{1}\right] \frac{i}{\hbar} \mu(\hat{Q})^{\times} \hat{\rho}_{L-1}^{\mathrm{eq}}\right\} .
$$

Here, $\operatorname{Tr}_{A}\{\cdots\}$ means the trace over the system $A$ 's states; $\hat{G}_{J K}[s]$ is expressed as (see Fig. 2)

$$
\hat{G}_{J K}[s]=\sum_{L=1}^{\min (J, K)}\left\{\frac{(J-1) !}{(L-1) !} \prod_{\alpha=L}^{J-1}\left(\hat{Z}_{J-\alpha+L-1}[s](-\hat{\Theta})\right) \cdot \hat{Z}_{L-1}[s] \cdot \prod_{\beta=L}^{K-1}\left((-\hat{\Phi}) \hat{Z}_{\beta}[s]\right)\right\},
$$

with

$$
\hat{Z}_{N}[s]=\frac{1}{s+i \hat{\mathcal{L}}_{A}+N \gamma-\hat{\Phi} \frac{N+1}{s+i \hat{\mathcal{L}}_{A}+(N+1) \gamma-\hat{\Phi} \frac{N+2}{s+i \hat{\mathcal{L}}_{A}+(N+2) \gamma-\cdots}} \hat{\Theta}},
$$

where for any operand operator $\hat{f}$,

$$
\begin{aligned}
& i \hat{\mathcal{L}}_{A} \hat{f} \equiv \frac{i}{\hbar} \hat{H}_{A} \times \hat{f} \\
& \hat{\Phi} \hat{f} \equiv \frac{i}{\hbar} V(\hat{Q})^{\times} \hat{f}, \\
& \hat{\Theta} \hat{f} \equiv \frac{i \zeta \gamma}{2}\left[i\left(\frac{\partial V(\hat{Q})}{\partial \hat{Q}} \hat{P} \hat{f}+\hat{f} \hat{P} \frac{\partial V(\hat{Q})}{\partial \hat{Q}}\right)+\frac{2 M}{\beta \hbar} V(\hat{Q})^{\times} \hat{f}\right]
\end{aligned}
$$

and for the system $A$ 's thermal density operator $\hat{\rho}_{A}^{(0)}$,

$$
\hat{\rho}_{L-1}^{\mathrm{eq}} \equiv \lim _{s \rightarrow 0} \hat{G}_{L 1}[s] \hat{\rho}_{A}^{(0)} .
$$

In the above, we set the following conditions for $N<L$ :

$$
\begin{aligned}
& \prod_{\alpha=L}^{N}\left(\hat{Z}_{J-\alpha+L-1}[s](-\hat{\Theta})\right)=1, \\
& \prod_{\beta=L}^{N}\left((-\hat{\Phi}) \hat{Z}_{\beta}[s]\right)=1 .
\end{aligned}
$$

The operators $\hat{\Theta}$ and $\hat{\Phi}$ have appeared corresponding to the phonon creation and annihilation processes, respectively. Thus the element $\hat{\rho}_{N}^{\text {eq }}$ represents the $N$-phonon dressed equilibrium state.
Although we are not dealing with the Gaussian-white noise bath characterized by the Ohmic spectral distribution,

$$
J^{\mathrm{GW}}(\omega)=\frac{M \zeta}{\pi} \omega\left(=\lim _{\gamma \rightarrow \infty} J^{\mathrm{GM}}(\omega)\right)
$$

it is worth a mention for later reference. The reduced response function with the Gaussian-white noise bath can be derived by following the procedure described in Appendix A, but can be quickly obtained by taking the limit, as $\gamma$ tends to infinity, of Eqs. (23)-(28). Then we have

$$
\begin{aligned}
R_{\mathrm{IR}}^{(3)}\left[s_{3}, s_{2}, s_{1}\right]= & \operatorname{Tr}_{A}\left\{\mu(\hat{Q}) \hat{\mathcal{G}}_{\mathrm{W}}\left[s_{3}\right] \frac{i}{\hbar} \mu(\hat{Q})^{\times} \hat{\mathcal{G}}_{\mathrm{W}}\left[s_{2}\right]\right. \\
& \left.\times \frac{i}{\hbar} \mu(\hat{Q})^{\times} \hat{\mathcal{G}}_{\mathrm{W}}\left[s_{1}\right] \frac{i}{\hbar} \mu(\hat{Q})^{\times} \hat{\rho}_{\mathrm{W}}^{\mathrm{eq}}\right\},
\end{aligned}
$$

with

$$
\begin{aligned}
& \hat{\mathcal{G}}_{\mathrm{W}}[s]=\lim _{\gamma \rightarrow \infty} \hat{G}_{11}[s]=\frac{1}{s+i \hat{\mathcal{L}}_{A}+\hat{\Gamma}_{\mathrm{W}}}, \\
& \hat{\rho}_{\mathrm{W}}^{\mathrm{eq}}=\lim _{s \rightarrow 0} \hat{\mathcal{G}}_{\mathrm{W}}[s] \hat{\rho}_{A}^{(0)},
\end{aligned}
$$

where $\hat{\Gamma}_{\mathrm{W}}$ is the relaxation operator given by 

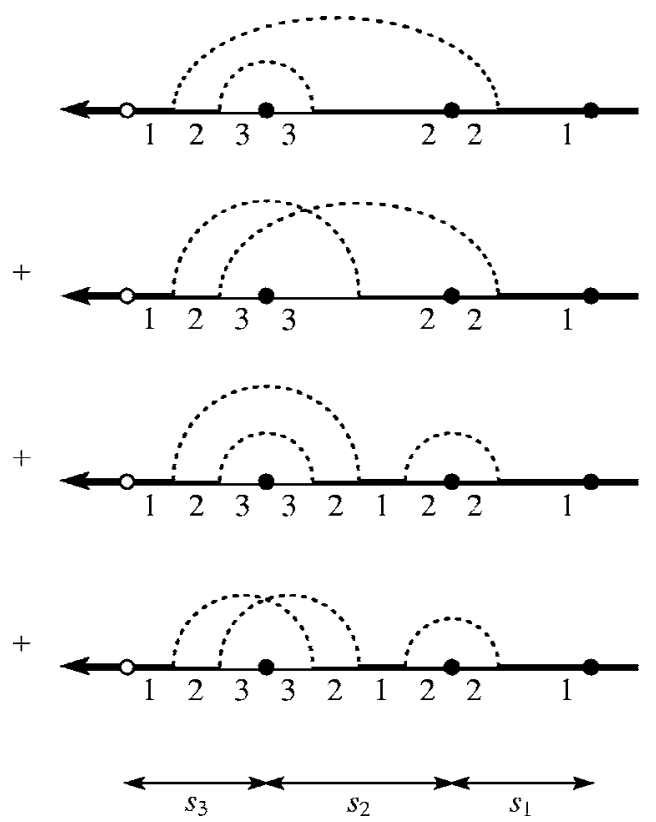

FIG. 3. Diagrammatic expression for the term of $(J, K, L)=(3,2,1)$ in Eq. (23). Here, we have used the diagrammatic expression for $\hat{G}_{13}[s], \hat{G}_{32}[s]$, and $\hat{G}_{21}[s]$ in Fig. 2. The closed circle stands for the interaction with a pulse: $\cdot=i / \hbar \cdot \mu(\hat{Q})^{\times}$, while the open circle represents the dipole radiation to be observed: ${ }^{\circ}=\mu(\hat{Q})$.

$$
\hat{\Gamma}_{\mathrm{W}}=-\lim _{\gamma \rightarrow \infty} \hat{\Phi} \frac{1}{\gamma} \hat{\Theta}
$$

The Gaussian-white noise bath is the motional narrowing (fast modulation) limit of the Gaussian-Markovian noise bath. In this limit, the contribution of quantum coherences to Eq. (23) is completely destroyed by the very fast fluctuation, which causes the reduction of Eq. (23) to Eq. (33).

\section{B. Two-state approximation}

For any physical operator $\hat{\mathcal{O}}$, we consider the norm $\|\hat{\mathcal{O}}\|$, which represents the magnitude of $\hat{\mathcal{O}}$. Then,

$$
\Delta \equiv \sqrt{\|\hat{\Phi}\| \cdot\|\hat{\Theta}\|}
$$

corresponds to the amplitude of the fluctuation. ${ }^{68,70}$ When the condition,

$$
\Delta \ll \gamma, \omega_{c}
$$

is satisfied, the deeper stages of the continued fraction, Eq. (25), can be ignored. Here, $\omega_{c}$ is the characteristic frequency of the system $A$. Note that the condition, Eq. (38), secures the Gaussian property of the bath noise. But we need not assume $\gamma$ so large compared with $\Delta$, because the continued fraction, Eq. (25), converges quickly even for not so large $\gamma$. As the result, we can approximately express the reduced response function, Eq. (23), as

$$
\begin{aligned}
R_{\mathrm{IR}}^{(3)}\left[s_{3}, s_{2}, s_{1}\right] \approx & \sum_{J, K, L=1}^{2} \operatorname{Tr}_{A}\left\{\mu(\hat{Q}) \hat{\mathcal{G}}_{1 J}\left[s_{3}\right] \frac{i}{\hbar} \mu(\hat{Q})^{\times} \hat{\mathcal{G}}_{J K}\left[s_{2}\right]\right. \\
& \left.\times \frac{i}{\hbar} \mu(\hat{Q})^{\times} \hat{\mathcal{G}}_{K L}\left[s_{1}\right] \frac{i}{\hbar} \mu(\hat{Q})^{\times} \hat{\rho}_{L-1}^{\mathrm{eq}}\right\},
\end{aligned}
$$

where

$$
\begin{aligned}
& \hat{\mathcal{G}}_{11}[s]=\frac{1}{s+i \hat{\mathcal{L}}_{A}-\hat{\Phi} \frac{1}{s+i \hat{\mathcal{L}}_{A}+\gamma} \hat{\Theta}}, \\
& \hat{\mathcal{G}}_{12}[s]=\hat{\mathcal{G}}_{11}[s](-\hat{\Phi}) \frac{1}{s+i \hat{\mathcal{L}}_{A}+\gamma}, \\
& \hat{\mathcal{G}}_{21}[s]=\frac{1}{s+i \hat{\mathcal{L}}_{A}+\gamma}(-\hat{\Theta}) \hat{\mathcal{G}}_{11}[s],
\end{aligned}
$$

and

$$
\begin{aligned}
\hat{\mathcal{G}}_{22}[s]= & \frac{1}{s+i \hat{\mathcal{L}}_{A}+\gamma}+\frac{1}{s+i \hat{\mathcal{L}}_{A}+\gamma}(-\hat{\Theta}) \hat{\mathcal{G}}_{11}[s] \\
& \times(-\hat{\Phi}) \frac{1}{s+i \hat{\mathcal{L}}_{A}+\gamma} .
\end{aligned}
$$

This approximation, Eq. (39), corresponds to taking into account only two of the bath states; namely, the phonon vacuum state and one-phonon excitation state. The condition, Eq. (38), can be removed if we regard our bath system as the noise source of the two-state-jump stochastic model, ${ }^{70,71}$ although here we included the temperature correction term which the original stochastic theory does not have. ${ }^{72,73}$

\section{TWO-DIMENSIONAL IR SIGNAL FROM THE DISSIPATIVE TUNNELING SYSTEM}

In this section, we present 2D IR signals for the dissipative tunneling process of the proton transfer system.

As an example where the condition of the nonadiabatic limit, Eq. (5), holds at the room temperature, we consider the case $Q_{0}=0.76 a_{0}$ and $U_{0}=2500 \mathrm{~cm}^{-1}$. These parameters give rise to one tunneling doublet (the splitting frequency $\omega_{10}$ $=20 \mathrm{~cm}^{-1}$ ) as shown in Fig. 1, where we have set $\omega_{21}$ $=2000 \mathrm{~cm}^{-1}$ and $\omega_{30}=3000 \mathrm{~cm}^{-1}$ for clarity. Here, we will calculate the signals over the frequency range as follows:

$$
\omega_{21} \lesssim \Omega \lesssim \omega_{30} \text {. }
$$

Then, assuming that the dipole moment $\mu(Q)$ of this system is a linear function of $Q$, i.e., $\mu(Q)=\mu_{0} Q$, the dipole operator $\mu(\hat{Q})$ can be expressed as

$$
\mu(\hat{Q}) \approx \mu_{0} \sum_{j=0,1} \sum_{k=0,1} Q_{2 j+1,2 k}(|2 j+1\rangle\langle 2 k|+| 2 k\rangle\langle 2 j+1|),
$$

where we have used abbreviated notation, $\mathcal{O}_{j k} \equiv\langle j|\hat{\mathcal{O}}| k\rangle$ for any operator $\hat{\mathcal{O}}$, and have used the fact that $Q_{2 j-1,2 j-1}$ and $Q_{2 k, 2 k}$ vanish due to the symmetry of potential. In addition, it 


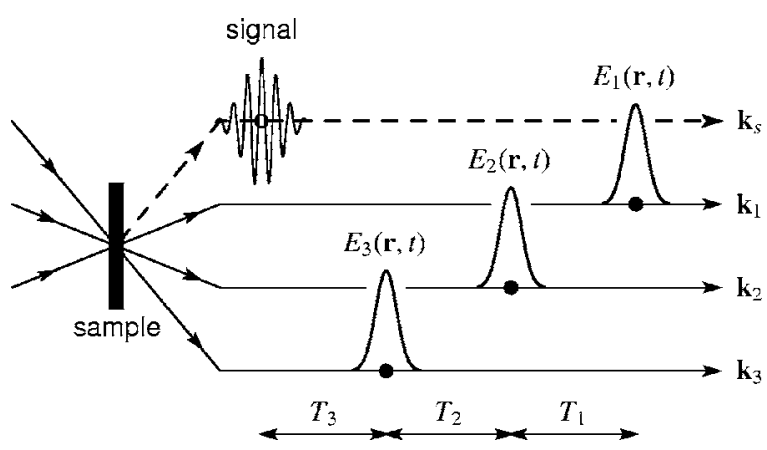

FIG. 4. Pulse configuration for the third-order IR experiment. The system interacts first with the pulse $E_{1}(\mathbf{r}, t)$, then with $E_{2}(\mathbf{r}, t)$, and finally with $E_{3}(\mathbf{r}, t) . T_{1}, T_{2}$, and $T_{3}$ represent the first, second, and third delay times, respectively.

is reasonable to assume that there is no bath-induced transition between the levels except for between $|0\rangle$ and $|1\rangle$ because $\exp \left(-\beta \hbar\left|\omega_{j k}\right|\right) \ll 1$ holds for $j \geqslant 2$ and $j \neq k$ even at the room temperature. Therefore, we can express $V(\hat{Q})$ in Eq. (8) as

$$
\begin{aligned}
V(\hat{Q}) & =K_{\mathrm{LL}} \sum_{j, k} Q_{j k}|j\rangle\left\langle k\left|+\frac{K_{\mathrm{SL}}}{2} \sum_{j, k} Q_{j k}^{2}\right| j\right\rangle\langle k| \\
& \approx K_{\mathrm{LL}} Q_{10}(|1\rangle\langle 0|+| 0\rangle\langle 1|)+\frac{K_{\mathrm{SL}}}{2} \sum_{j} Q_{j j}^{2}|j\rangle\langle j| .
\end{aligned}
$$

From Eq. (46), we can show $\hat{\rho}_{N}^{\mathrm{eq}}$ for $N \geqslant 1$.

Equations (45) and (46) enable us to treat the system $A$ characterized by the symmetric double-well potential, Eq. (2), as a four-level system ${ }^{71}$ which consists of $|0\rangle,|1\rangle,|2\rangle$, and $|3\rangle$; in the Liouville space, any operator can be represented by a $4^{2} \times 4^{2}$ matrix in the $\{|j\rangle\langle k|\}_{0 \leqslant j, k \leqslant 3}$ basis. Note that for $2 \mathrm{D}$-IR spectroscopy, it is usually necessary to consider energy levels higher than $|3\rangle$ due to the excitations with two vibrational quanta. Spectral peaks which arise from the processes with two vibrational quanta are, however, almost time independent and may appear as independent peaks. Since their contributions are easily separated from others and are not essential for the following discussion, here we leave such contributions out and we restrict our discussion only to the four-level system.

\section{A. Direct observation of the thermal excitation and relaxation processes}

Consider the laser field at the position $\mathbf{r}$ consists of three pulses,

$$
E(\mathbf{r}, t)=\sum_{j=1}^{3} E_{j}(\mathbf{r}, t)
$$

with

$$
E_{j}(\mathbf{r}, t)=\overline{E_{j}}(t)\left(e^{i \mathbf{k}_{j} \cdot \mathbf{r}-i \omega_{j} t}+\text { c.c. }\right) .
$$

Here, $\mathbf{k}_{j}$ and $\omega_{j}$ are the wave vector and frequency, respectively, and $\bar{E}_{j}(t)$ is the temporal envelope of the $j$ th incident pulse.
Although one should appropriately treat colors of laser fields ${ }^{74,75}$ and their effects on a phase matching condition for nonlinear IR measurement, ${ }^{76}$ here we consider the impulsive limit of the pulses expressed as (see Fig. 4)

$$
\begin{aligned}
& \overline{E_{1}}(t)=\delta\left(t-\left(t_{m}-T_{3}-T_{2}-T_{1}\right)\right), \\
& \overline{E_{2}}(t)=\delta\left(t-\left(t_{m}-T_{3}-T_{2}\right)\right), \\
& \overline{E_{3}}(t)=\delta\left(t-\left(t_{m}-T_{3}\right)\right),
\end{aligned}
$$

for demonstration purpose, where $\delta(t)$ is the Dirac delta function. Then, the polarization, Eq. (17), in the direction $\mathbf{k}_{s}=\mathbf{k}_{1}-\mathbf{k}_{2}+\mathbf{k}_{3}$ is given by

$$
P^{(3)}\left(\mathbf{r}, t_{m}\right)=e^{i \mathbf{k}_{s} \cdot \mathbf{r}-i \omega_{s} t_{m}} P_{\mathbf{k}_{s}}^{(3)}
$$

with $\omega_{s}=\omega_{1}-\omega_{2}+\omega_{3}$ and

$$
P_{\mathbf{k}_{s}}^{(3)}=e^{i\left(\omega_{3}-\omega_{2}+\omega_{1}\right) T_{3}} e^{-i\left(\omega_{2}-\omega_{1}\right) T_{2}} e^{i \omega_{1} T_{1}} \mathcal{R}_{\mathrm{IR}}^{(3)}\left(T_{3}, T_{2}, T_{1}\right) .
$$

Here, we assume that the frequencies of the three incident pulses are tuned to $\left(\omega_{21}+\omega_{30}\right) / 2=2500 \mathrm{~cm}^{-1}$. Then, the response function $\mathcal{R}_{\mathrm{IR}}^{(3)}\left(T_{3}, T_{2}, T_{1}\right)$ is given as the sum of the Liouville space pathways represented by the following double-sided Feynman diagrams: ${ }^{15}$

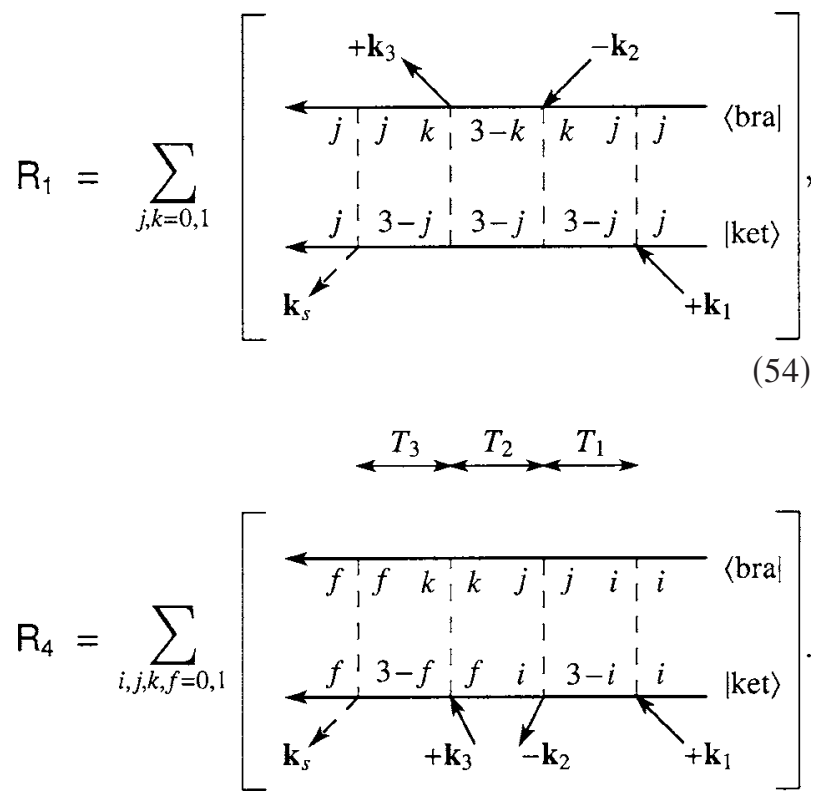

In these diagrams, the lower horizontal lines correspond to the time evolution (from the right to the left) of the ket and the upper lines to that of the bra. The oblique arrows stand for the laser interactions. The diagram, Eq. (54), can be interpreted as follows. At first the system is in the population state $|j\rangle\langle j|(j=0,1)$. At $t=t_{m}-T_{3}-T_{2}-T_{1}$, the ket $|j\rangle$ interacts with the first laser $+\mathbf{k}_{1}$, and the system is changed into the coherence state $|3-j\rangle\langle j|$. During the time interval $T_{1}$, the coupling with the bath may change the bra $\langle j|$ into $\langle k|(k=0$, 1 and $k \neq j$ ). This process is referred to as bath-induced coherence transfer $|3-j\rangle\langle j|\rightarrow| 3-j\rangle\langle k|(k \neq j)$. At $t=t_{m}-T_{3}$ $-T_{2}$, the bra $\langle k|$ interacts with the second laser $-\mathbf{k}_{2}$, and the system is in the state $|3-j\rangle\langle 3-k|$ in the next period $T_{2}$. The 


\section{(a) $\omega_{10} T_{2}=0$}

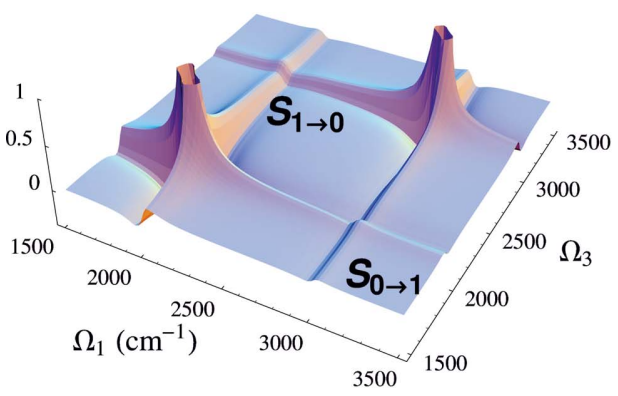

(c) $\omega_{10} T_{2}=0.4$

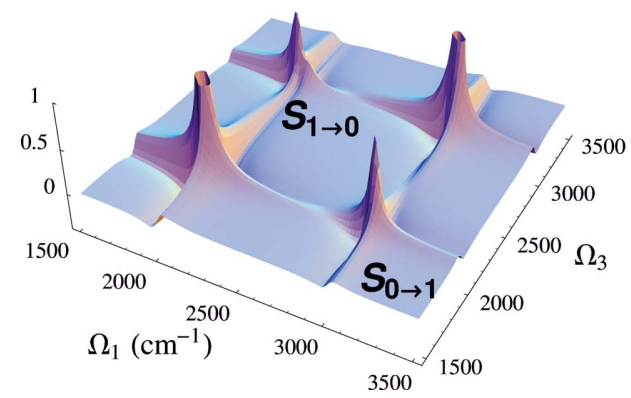

(b) $\omega_{10} T_{2}=0.1$

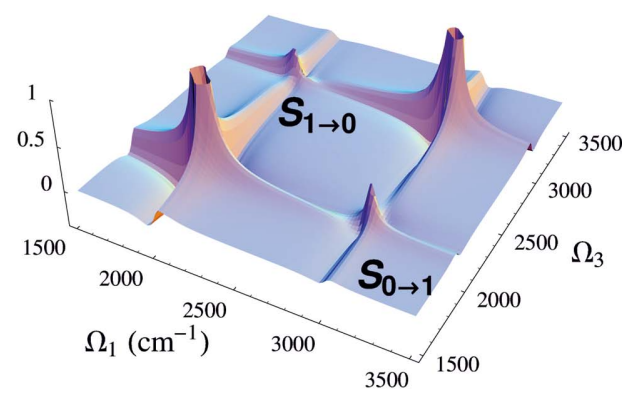

(d) $\omega_{10} T_{2}=3$

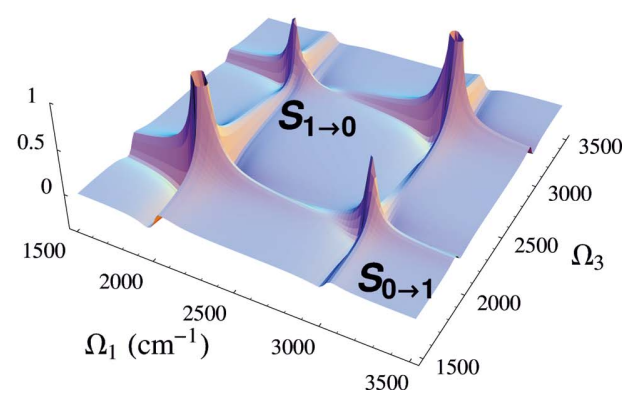

FIG. 5. (Color) Two-dimensional IR signals $S\left(\Omega_{1}, \Omega_{3} ; T_{2}\right)$ calculated for the system in Fig. 1 under the two-state-jump approximation. The relevant transition frequencies are $\omega_{21}=2000 \mathrm{~cm}^{-1}, \omega_{30}=3000 \mathrm{~cm}^{-1}$, and $\omega_{10}=20 \mathrm{~cm}^{-1}\left(1 / \omega_{10}=1.66 \mathrm{ps}\right)$. The system-bath parameters are $\zeta_{L L}=2 \omega_{10}, \zeta_{S L}{ }^{\prime}=1, \gamma=6 \omega_{10}$, and $\beta$ $=2.41 \times 10^{20}(300 \mathrm{~K})$. The normalization of each graph is such that the maximum value of the signal observed at $\left(\Omega_{1}, \Omega_{3}\right)=(3000,2000) \mathrm{cm}^{-1}$ is unity. The three incident pulses are assumed to be tuned to $2500 \mathrm{~cm}^{-1}$.

subsequent events can be interpreted correspondingly. Although Eq. (55) can be interpreted in the same way, note that there appear not only coherence transfer processes but also bath-induced conversion from coherence to population processes $|i\rangle\langle j|\rightarrow| f\rangle\langle k|(i \neq j$ and $f=k)$ and vice versa $|i\rangle\langle j|$ $\rightarrow|f\rangle\langle k|(i=j$ and $f \neq k)$ during the $T_{2}$ period.

The 2D-IR signal to be discussed below is the imaginary part of the two-dimensional Fourier transform of $-\mathcal{R}_{\mathrm{IR}}^{(3)}\left(T_{3}, T_{2}, T_{1}\right)$ with respect to $T_{1}$ and $T_{3}$,

$$
S\left(\Omega_{1}, \Omega_{3} ; T_{2}\right)=\operatorname{Im}\left[-\mathcal{R}_{\mathrm{IR}}^{(3)}\left(\Omega_{3}, T_{2}, \Omega_{1}\right)\right],
$$

with

$$
\begin{aligned}
\mathcal{R}_{\mathrm{IR}}^{(3)}\left(\Omega_{3}, T_{2}, \Omega_{1}\right) & =\int_{0}^{\infty} d T_{3} \int_{0}^{\infty} d T_{1} e^{i \Omega_{3} T_{3}+i \Omega_{1} T_{1}} \mathcal{R}_{\mathrm{IR}}^{(3)}\left(T_{3}, T_{2}, T_{1}\right) \\
& =\frac{1}{2 \pi i} \int_{c-i \infty}^{c+i \infty} d s_{2} e^{s_{2} T_{2}} \mathcal{R}_{\mathrm{IR}}^{(3)}\left[-i \Omega_{3}, s_{2},-i \Omega_{1}\right],
\end{aligned}
$$

where the constant $c$ is chosen in such a way that all singular points of the integrand lie on the left side of the path.

Figure 5 shows the three-dimensional plots of $S\left(\Omega_{1}, \Omega_{3} ; T_{2}\right)$ calculated under the two-state-jump approximation as a function of $\Omega_{1}$ and $\Omega_{3}$ at the temperature $300 \mathrm{~K}$ for (a) $\omega_{10} T_{2}=0$, (b) $\omega_{10} T_{2}=0.1$, (c) $\omega_{10} T_{2}=0.4$, and (d) $\omega_{10} T_{2}=3$, where $1 / \omega_{10}=1.66 \mathrm{ps}$ for $\omega_{10}=20 \mathrm{~cm}^{-1}$. The LL coupling strength, the SL coupling strength, and the correlation time of the bath noise are, respectively, chosen to be

$$
\zeta_{\mathrm{LL}}=2 \omega_{10}, \quad \zeta_{\mathrm{SL}}{ }^{\prime}=1, \quad \tau_{c}=\frac{1}{\gamma}=\frac{1}{6 \omega_{10}}
$$

where we have introduced the dimensionless SL coupling strength defined by $\zeta_{S L}{ }^{\prime}=\hbar \zeta_{S L} /\left(M \omega_{10}{ }^{2}\right)$. In Fig. 5, there appear the cross peaks $S_{0 \rightarrow 1}$ and $S_{1 \rightarrow 0}$ around at $\left(\Omega_{1}, \Omega_{3}\right)$ $=\left(\omega_{30}, \omega_{21}\right)$ and $\left(\omega_{21}, \omega_{30}\right)$, which are not observed initially. These peaks grow with time. Here, note that these peaks come from the terms of $(i, f)=(0,1)$ and $(1,0)$ in Eq. (55). Then the peak intensities, $S_{0 \rightarrow 1}\left(\Omega_{1}, \Omega_{3} ; T_{2}\right)$ and $S_{1 \rightarrow 0}\left(\Omega_{1}, \Omega_{3} ; T_{2}\right)$, are expressed as follows (see Fig. 6):

$$
\begin{aligned}
S_{0 \rightarrow 1}\left(\Omega_{1}, \Omega_{3} ; T_{2}\right)= & C \sum_{J, K=1}^{\infty} \sum_{j, k=0,1} \operatorname{Re}\left[\left\langle\left\langle 21\left|\hat{G}_{1 J}\left[-i \Omega_{3}\right]\right| 2 j\right\rangle\right\rangle\right. \\
& \times\left\langle\left\langle 1 j\left|\hat{G}_{J K}\left(T_{2}\right)\right| 0 k\right\rangle\right\rangle \\
& \left.\times\left\langle\left\langle 3 k\left|\hat{G}_{K 1}\left[-i \Omega_{1}\right]\right| \mid 30\right\rangle\right\rangle\right]\left(\rho_{0}^{\mathrm{eq}}\right)_{00}
\end{aligned}
$$

and

$$
\begin{aligned}
S_{1 \rightarrow 0}\left(\Omega_{1}, \Omega_{3} ; T_{2}\right)= & C \sum_{J, K=1}^{\infty} \sum_{j, k=0,1} \operatorname{Re}\left[\left\langle\left\langle 30\left|\hat{G}_{1 J}\left[-i \Omega_{3}\right]\right| 3 j\right\rangle\right\rangle\right. \\
& \times\left\langle\left\langle 0 j\left|\hat{G}_{J K}\left(T_{2}\right)\right| 1 k\right\rangle\right\rangle \\
& \left.\times\left\langle\left\langle 2 k\left|\hat{G}_{K 1}\left[-i \Omega_{1}\right]\right| 21\right\rangle\right\rangle\right]\left(\rho_{0}^{\mathrm{eq}}\right)_{11}, \quad(60)
\end{aligned}
$$

with 
(a)

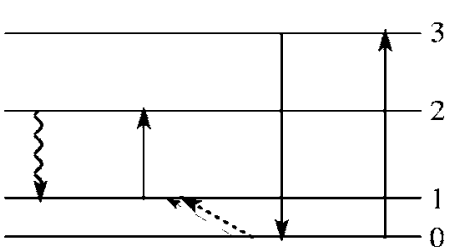

(b)
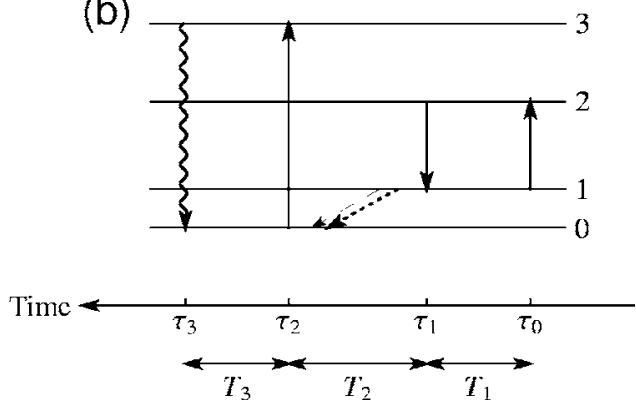

FIG. 6. Energy-level diagrams contributing to the cross peaks. The diagram (a) involving the excitation $0 \rightarrow 1$ is for the signal $S_{0 \rightarrow 1}\left(\Omega_{1}, \Omega_{3} ; T_{2}\right)$; the diagram (b) involving the relaxation $1 \rightarrow 0$ is for the signal $S_{1 \rightarrow 0}\left(\Omega_{1}, \Omega_{3} ; T_{2}\right)$.

$$
C \equiv \frac{\mu_{0}^{4} Q_{30}^{2} Q_{21}^{2}}{\hbar^{3}}
$$

where $\hat{G}_{J K}(t)$ is the inverse Laplace transform of $\hat{G}_{J K}[s]$. In the above, we have introduced the Liouville space vector $|j k\rangle\rangle$ representing a Hilbert space operator $|j\rangle\langle k|$. The Hermitian conjugate to $|m n\rangle\rangle$ is denoted by $\langle\langle m n|$; a scalar product is defined as $\langle\langle j k \mid m n\rangle\rangle=\operatorname{Tr}[|k\rangle\langle j \mid m\rangle\langle n|]$. This behavior of the signal $S\left(\Omega_{1}, \Omega_{3} ; T_{2}\right)$ is due principally to the thermal transition of the population between the tunneling doublet $|0\rangle\langle 0|\leftrightarrow| 1\rangle\langle 1|$. To illustrate this, we consider the 2D-IR signal with the Gaussian-white noise bath in the absence of the SL coupling term, i.e., $K_{\mathrm{LL}}=1$ and $K_{\mathrm{SL}}=0$. In this case, using Eqs. (33)-(36), we can obtain the simple expressions for $S_{0 \rightarrow 1}\left(\Omega_{1}, \Omega_{3} ; T_{2}\right)$ and $S_{1 \rightarrow 0}\left(\Omega_{1}, \Omega_{3} ; T_{2}\right)$,

$$
S_{0 \rightarrow 1}^{\mathrm{w}}\left(\Omega_{1}, \Omega_{3} ; T_{2}\right)=\frac{C\left\langle\left\langle 11\left|\hat{\mathcal{G}}_{\mathrm{w}}\left(T_{2}\right)\right| 00\right\rangle\right\rangle}{\operatorname{Re}\left[F^{(30)}\left(\Omega_{1}\right) F^{(21)}\left(\Omega_{3}\right)\right]}\left(\rho_{\mathrm{w}}^{\mathrm{eq}}\right)_{00},
$$

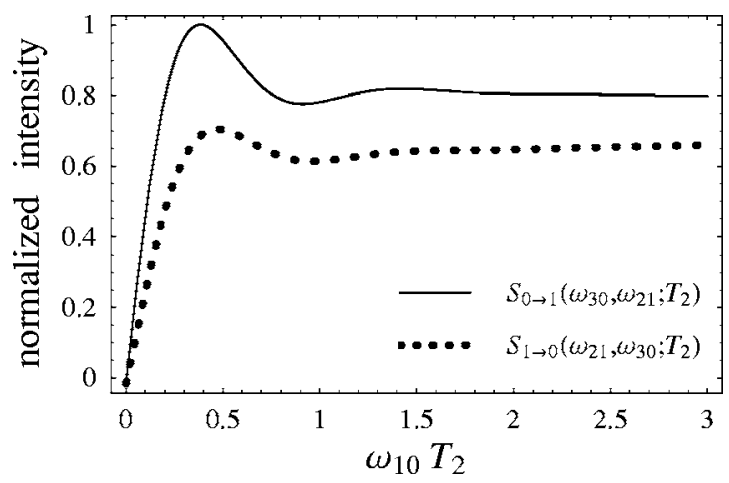

FIG. 7. Total intensity of the signals as the function of time $T_{2}$ given in Fig. 5 , where $1 / \omega_{10}=1.66 \mathrm{ps}$ for $\omega_{10}=20 \mathrm{~cm}^{-1}$. The solid line represents the total intensity $S_{0 \rightarrow 1}\left(\omega_{30}, \omega_{21} ; T_{2}\right)$, which corresponds to the transition $|0\rangle\langle 0|$ $\rightarrow|1\rangle\langle 1|$; the dotted line represents the total intensity of $S_{1 \rightarrow 0}\left(\omega_{21}, \omega_{30} ; T_{2}\right)$, which corresponds to $|1\rangle\langle 1|\rightarrow| 0\rangle\langle 0|$. Since there are contributions from $\mathrm{CP}$ and CCP processes explained in Fig. 8, two lines do not agree.

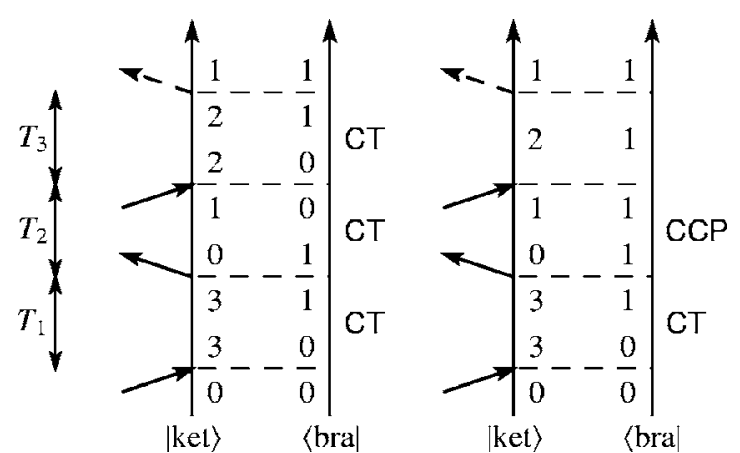

FIG. 8. Examples of the double-sided Feynman diagrams which represent the bath-induced coherence transfer (CT) and the bath-induced conversion from coherence to population (CCP) contributing to $S_{0 \rightarrow 1}\left(\omega_{30}, \omega_{21} ; T_{2}\right)$.

$$
S_{1 \rightarrow 0}^{\mathrm{w}}\left(\Omega_{1}, \Omega_{3} ; T_{2}\right)=\frac{C\left\langle\left\langle 00\left|\hat{\mathcal{G}}_{\mathrm{w}}\left(T_{2}\right)\right| 11\right\rangle\right\rangle}{\operatorname{Re}\left[F^{(21)}\left(\Omega_{1}\right) F^{(30)}\left(\Omega_{3}\right)\right]}\left(\rho_{\mathrm{w}}^{\mathrm{eq}}\right)_{11},
$$

with the transition probabilities,

$$
\begin{aligned}
& \left\langle\left\langle 11\left|\hat{\mathcal{G}}_{\mathrm{w}}(t)\right| 00\right\rangle\right\rangle=\left(1-e^{-2 \alpha \zeta^{\zeta} t}\right) n_{-}, \\
& \left\langle\left\langle 00\left|\hat{\mathcal{G}}_{\mathrm{w}}(t)\right| 11\right\rangle\right\rangle=\left(1-e^{-2 \alpha \zeta^{\zeta} t}\right) n_{+},
\end{aligned}
$$

and the matrix elements of $\hat{\rho}_{\mathrm{w}}^{\mathrm{eq}}$,

$$
\left(\rho_{\mathrm{w}}^{\mathrm{eq}}\right)_{00}=n_{+}, \quad\left(\rho_{\mathrm{w}}^{\mathrm{eq}}\right)_{11}=n_{-},
$$

where we have set

$$
\begin{aligned}
& F^{(30)}(\Omega) \equiv i\left(\Omega-\omega_{30}\right)-\alpha \zeta n_{-}, \\
& F^{(21)}(\Omega) \equiv i\left(\Omega-\omega_{21}\right)-\alpha \zeta n_{+},
\end{aligned}
$$

and

$$
\alpha \equiv \frac{2 M Q_{10}^{2}}{\beta \hbar^{2}}, \quad n_{ \pm} \equiv \frac{2 \pm \beta \hbar \omega_{10}}{4} .
$$

From Eqs. (62) and (63), we obtain the intensities of the peaks:

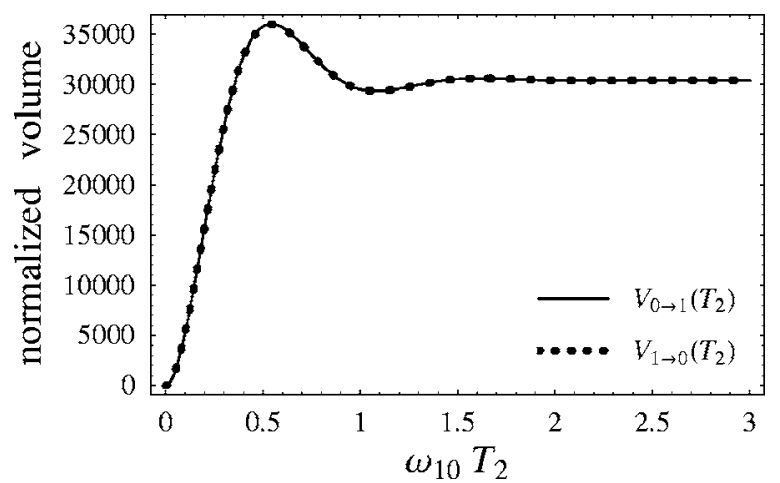

FIG. 9. The volume of the peak in Fig. 5 is plotted as the function of the time $T_{2}$, where $1 / \omega_{10}=1.66 \mathrm{ps}$ for $\omega_{10}=20 \mathrm{~cm}^{-1}$. The solid line is for the peak of $S_{0 \rightarrow 1}\left(\Omega_{1}, \Omega_{3} ; T_{2}\right)$, which corresponds to the excitation $|0\rangle\langle 0|\rightarrow| 1\rangle$ $\times\langle 1|$, whereas the dotted line is for the peak of $S_{1 \rightarrow 0}\left(\Omega_{1}, \Omega_{3} ; T_{2}\right)$, which corresponds to $|1\rangle\langle 1|\rightarrow| 0\rangle\langle 0|$. By observing the volume of the cross peaks instead of the total intensity of the signals, we can separate the CT and CPP contributions from the net transition between the states. 


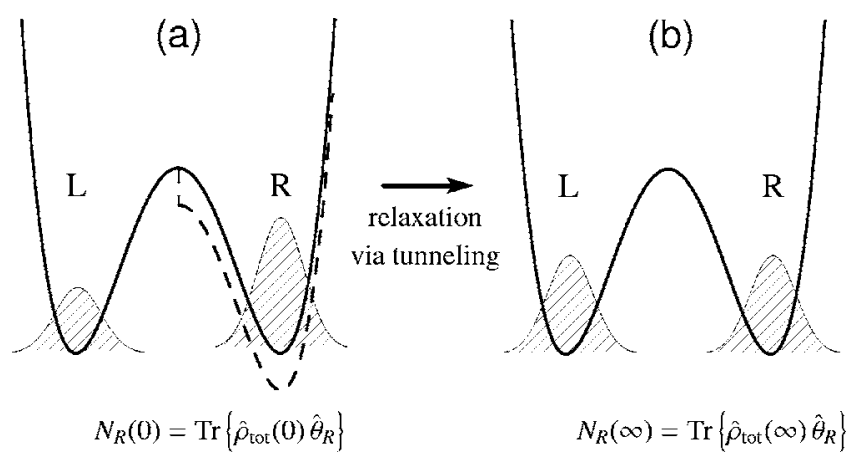

FIG. 10. The unperturbed (solid line) and perturbed potentials (dashed line). The population equilibrated with the perturbation $N_{R}(0)$ relaxes to those of the unperturbed system $N_{R}(\infty)$ via tunneling with the relaxation rate $k(t)$.

$$
\begin{aligned}
& S_{0 \rightarrow 1}^{\mathrm{w}}\left(\omega_{30}, \omega_{21} ; T_{2}\right)=\frac{C}{\alpha^{2} \zeta^{2} n_{+} n_{-}}\left\langle\left\langle 11\left|\hat{\mathcal{G}}_{\mathrm{w}}\left(T_{2}\right)\right| 00\right\rangle\right\rangle\left(\rho_{\mathrm{w}}^{\mathrm{eq}}\right)_{00}, \\
& S_{1 \rightarrow 0}^{\mathrm{w}}\left(\omega_{21}, \omega_{30} ; T_{2}\right)=\frac{C}{\alpha^{2} \zeta^{2} n_{+} n_{-}}\left\langle\left\langle 00\left|\hat{\mathcal{G}}_{\mathrm{w}}\left(T_{2}\right)\right| 11\right\rangle\right\rangle\left(\rho_{\mathrm{w}}^{\mathrm{eq}}\right)_{11},
\end{aligned}
$$

These results clearly show that the thermal excitation from $|0\rangle\langle 0|$ to $|1\rangle\langle 1|$ and the relaxation from $|1\rangle\langle 1|$ to $|0\rangle\langle 0|$ cause the growth of the cross peaks $S_{0 \rightarrow 1}^{\mathrm{w}}\left(\Omega_{1}, \Omega_{3} ; T_{2}\right)$ and $S_{1 \rightarrow 0}^{\mathrm{W}}\left(\Omega_{1}, \Omega_{3} ; T_{2}\right)$, respectively. An additional remark which should be made here is that both intensities are equal for any $T_{2}$,

$$
S_{0 \rightarrow 1}^{\mathrm{W}}\left(\omega_{30}, \omega_{21} ; T_{2}\right)=S_{1 \rightarrow 0}^{\mathrm{w}}\left(\omega_{21}, \omega_{30} ; T_{2}\right),
$$

which is the outcome of the detailed balance condition:

$$
\left\langle\left\langle 11\left|\hat{\mathcal{G}}_{\mathrm{w}}\left(T_{2}\right)\right| 00\right\rangle\right\rangle\left(\rho_{\mathrm{w}}^{\mathrm{eq}}\right)_{00}=\left\langle\left\langle 00\left|\hat{\mathcal{G}}_{\mathrm{w}}\left(T_{2}\right)\right| 11\right\rangle\right\rangle\left(\rho_{\mathrm{w}}^{\mathrm{eq}}\right)_{11} .
$$

Next, Fig. 7 gives the plots of $S_{0 \rightarrow 1}\left(\omega_{30}, \omega_{21} ; T_{2}\right)$ and $S_{1 \rightarrow 0}\left(\omega_{21}, \omega_{30} ; T_{2}\right)$ in Fig. 5 as a function of $T_{2}$. As is evident from the plots, the intensities of the peaks in our model are not equal:

$$
S_{0 \rightarrow 1}\left(\omega_{30}, \omega_{21} ; T_{2}\right) \neq S_{1 \rightarrow 0}\left(\omega_{21}, \omega_{30} ; T_{2}\right) .
$$

This is because the growth of the peaks for GaussianMarkovian noise bath case in the presence of the SL coupling is due to not only the thermal transition of the population, but also the other processes, i.e., the bath-induced coherence transfer $|j\rangle\langle k|\rightarrow| l\rangle\langle m|(j \neq k, l \neq m)$, and the bathinduced conversion from coherence to population $|j\rangle\langle k|$ $\rightarrow|n\rangle\langle n|(j \neq k)$, and vice versa ${ }^{33}$ (see Fig. 8). In the Gaussian-white noise case, these processes are completely destroyed by the very fast fluctuation as mentioned in the preceding section.

Although the peak intensities involve the contribution from the thermal transition of population and the coherence transfer, we can eliminate the later contribution by evaluating the peak volumes. In Fig. 9, we show the change of the volumes as the function of time $T_{2}: V_{0 \rightarrow 1}\left(T_{2}\right)$ and $V_{1 \rightarrow 0}\left(T_{2}\right)$ for the peaks of $S_{0 \rightarrow 1}\left(\Omega_{1}, \Omega_{3} ; T_{2}\right)$ and $S_{1 \rightarrow 0}\left(\Omega_{1}, \Omega_{3} ; T_{2}\right)$, respectively. Unlike the intensities, the two plots accord well together for any $T_{2}$. This accordance indicates that we have observed only the thermal transition of the population via the volume of the peaks; this can be proven without the assumption of the two-state-jump approximation in the following way. From Eq. (59), the volume of $S_{0 \rightarrow 1}\left(\Omega_{1}, \Omega_{3} ; T_{2}\right)$ is evaluated as

$$
\begin{aligned}
V_{0 \rightarrow 1}\left(T_{2}\right)= & \int_{-\infty}^{\infty} d \Omega_{1} \int_{-\infty}^{\infty} d \Omega_{3} S_{0 \rightarrow 1}\left(\Omega_{1}, \Omega_{3} ; T_{2}\right) \\
= & \lim _{T_{1}, T_{3} \rightarrow 0}(2 \pi)^{2} \int_{-\infty}^{\infty} \frac{d \Omega_{1}}{2 \pi} \int_{-\infty}^{\infty} \frac{d \Omega_{3}}{2 \pi} e^{-i \Omega_{1} T_{1}-i \Omega_{3} T_{3}} \\
& \times S_{0 \rightarrow 1}\left(\Omega_{1}, \Omega_{3} ; T_{2}\right) \\
= & (2 \pi)^{2} C \cdot\left\langle\left\langle 11\left|\hat{G}_{11}\left(T_{2}\right)\right| 00\right\rangle\right\rangle\left(\rho_{0}^{\mathrm{eq}}\right)_{00} .
\end{aligned}
$$

In a similar fashion, the volume of $S_{1 \rightarrow 0}\left(\Omega_{1}, \Omega_{3} ; T_{2}\right)$ is also calculated as

$$
V_{1 \rightarrow 0}\left(T_{2}\right)=(2 \pi)^{2} C \cdot\left\langle\left\langle 00\left|\hat{G}_{11}\left(T_{2}\right)\right| 11\right\rangle\right\rangle\left(\rho_{0}^{\mathrm{eq}}\right)_{11} .
$$

As is the case with Eq. (72), the detailed balance condition leads to the relation:

$$
V_{0 \rightarrow 1}\left(T_{2}\right)=V_{1 \rightarrow 0}\left(T_{2}\right) .
$$

Once we evaluate the contribution of thermal transition of population, we can easily estimate the contribution of the coherence transfer by comparing $V_{1 \rightarrow 0}\left(T_{2}\right)$ and $S_{1 \rightarrow 0}\left(T_{2}\right)$, etc. Consequently, the bath-induced vibrational excitation and relaxation dynamics can be separated from other processes and can be directly observed by means of two-dimensional spectroscopy with the present pulse configuration.

\section{B. The ability of the two-dimensional IR spectroscopy for evaluating reaction rate constants}

A chemical reaction rate is one of the most important observables for investigating chemical reaction processes. As is well known, however, it is not so easy to determine the rate experimentally because the rate constant is defined by a probability density and it is usually not a direct observable. We expect our multidimensional vibrational spectroscopy to cast a new light on this issue. In this section, we demonstrate the ability of the 2D-IR spectroscopy for evaluating the chemical reaction rate.

The rate constant $k_{\mathrm{rxn}}$ can be represented as the asymptotic limit of the Kubo-transformed reactive-flux correlation function ${ }^{4}$ (see Appendix C),

$$
k(t)=\frac{\left\langle\delta \dot{\hat{\theta}}_{R} ; \delta \hat{\theta}_{R}(t)\right\rangle}{\left\langle\delta \hat{\theta}_{R} ; \delta \hat{\theta}_{R}(t)\right\rangle},
$$

where $\hat{\theta}_{R}=\theta(\hat{Q})$ is the unit Heaviside function measuring the occupancy of the right side of the double well (see Fig. 10), $\delta \hat{\theta}_{R}=\hat{\theta}_{R}-\left\langle\hat{\theta}_{R}\right\rangle$, and $\delta \dot{\hat{\theta}}_{R}=\left[\delta \hat{\theta}_{R}, \hat{H}_{\text {tot }}\right] / i \hbar$. In the above, we have introduced the canonical correlation function ${ }^{77}$ for any two operators $\hat{F}$ and $\hat{G}$,

$$
\langle\hat{F} ; \hat{G}\rangle=\int_{0}^{\beta} \frac{d \lambda}{\beta}\left\{\hat{\rho}_{\mathrm{tot}}^{\mathrm{eq}} e^{\lambda \hat{H}_{\mathrm{tot}}} \hat{F} e^{-\lambda \hat{H}_{\mathrm{tot}}} \hat{G}\right\} .
$$




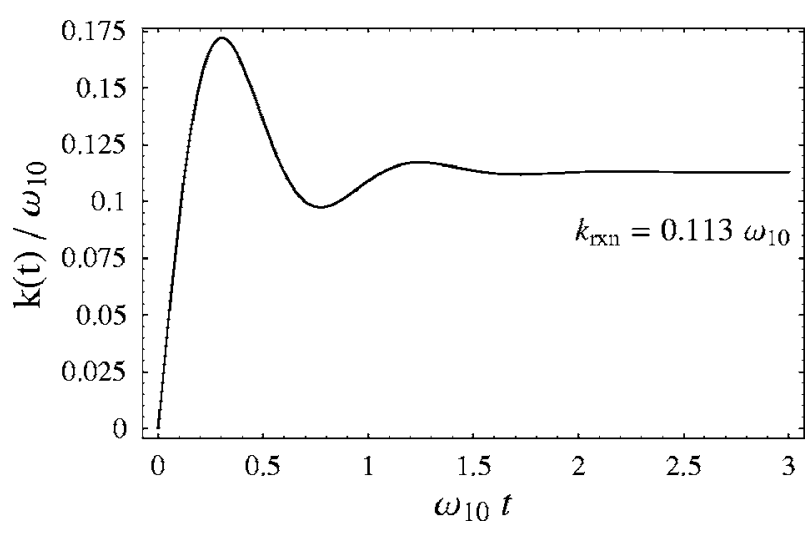

FIG. 11. Time evolution of the chemical reaction rate calculated from Eq (82) for the system-bath parameters $\zeta_{L L}=2.5 \omega_{10}, \zeta_{S L}{ }^{\prime}=0.5, \gamma=6 \omega_{10}$, and $\beta$ $=2.41 \times 10^{20}(300 \mathrm{~K})$, where $1 / \omega_{10}=1.66 \mathrm{ps}$ for $\omega_{10}=20 \mathrm{~cm}^{-1}$. After the transient behavior for short times, we see a plateau regime; $k(t)$ converges to a constant $k_{\mathrm{rxn}}=0.113 \omega_{10}$.

Tracing over the bath degrees of freedom from Eq. (78) leads us to

$$
k(t)=\frac{\sum_{J=1}^{\infty} \operatorname{Tr}_{A}\left\{\delta \hat{\theta}_{R} \hat{G}_{1 J}(t) \frac{i}{\hbar} \delta \hat{\theta}_{R}^{\times} \hat{\rho}_{J-1}^{\mathrm{eq}}\right\}}{\int_{t}^{\infty} d s \sum_{J=1}^{\infty} \operatorname{Tr}_{A}\left\{\delta \hat{\theta}_{R} \hat{G}_{1 J}(s) \frac{i}{\hbar} \delta \hat{\theta}_{R}^{\times} \hat{\rho}_{J-1}^{\mathrm{eq}}\right\}} .
$$

In addition, for the present model in the nonadiabatic limit, Eq. (5), $\hat{\theta}_{R}$ can be assumed to be of the form

$$
\hat{\theta}_{R}=\frac{|0\rangle+|1\rangle}{\sqrt{2}} \cdot \frac{\langle 0|+\langle 1|}{\sqrt{2}} .
$$

Consequently, the rate $k(t)$ for the nonadiabatic proton transfer reaction can be expressed as (see Fig. 11)

$$
k(t)=\frac{\operatorname{Im}\left[-\left\langle\left\langle 10\left|\hat{G}_{11}(t)\right| 10\right\rangle\right\rangle\right]}{\int_{t}^{\infty} d s \operatorname{Im}\left[-\left\langle\left\langle 10\left|\hat{G}_{11}(s)\right| 10\right\rangle\right\rangle\right]} .
$$

What has to be noticed here is that Eq. (82) cannot be applied for the case of coherent oscillatory motion, where the system-bath coupling is so weak that $\left\langle\left\langle 10\left|\hat{G}_{11}(t)\right| 10\right\rangle\right\rangle$ oscillates between positive and negative values due to the quantum coherence.

Now, we consider the laser field, Eqs. (47)-(51); the frequencies of the three incident pulses are tuned to $\omega_{1}$ $\sim \omega_{30}, \omega_{2} \sim \omega_{32}$, and $\omega_{3} \sim \omega_{21}$, respectively. The polarization detected in the direction $\mathbf{k}_{s}=\mathbf{k}_{1}-\mathbf{k}_{2}-\mathbf{k}_{3}$ is given by

$$
P^{(3)}\left(\mathbf{r}, t_{m}\right)=e^{i \mathbf{k}_{s} \cdot \mathbf{r}-i \omega_{s} t_{m}} P_{\mathbf{k}_{s}}^{(3)},
$$

with $\omega_{s}=\omega_{1}-\omega_{2}-\omega_{3}$ and

$$
P_{\mathbf{k}_{s}}^{(3)}=e^{i\left(-\omega_{3}-\omega_{2}+\omega_{1}\right) T_{3}} e^{-i\left(\omega_{2}-\omega_{1}\right) T_{2}} e^{i \omega_{1} T_{1}} \mathcal{R}_{I R}^{(3)}\left(T_{3}, T_{2}, T_{1}\right),
$$

where the response function $\mathcal{R}_{\mathrm{IR}}^{(3)}\left(T_{3}, T_{2}, T_{1}\right)$ is expressed by the double-sided Feynman diagram as (see Fig. 12)
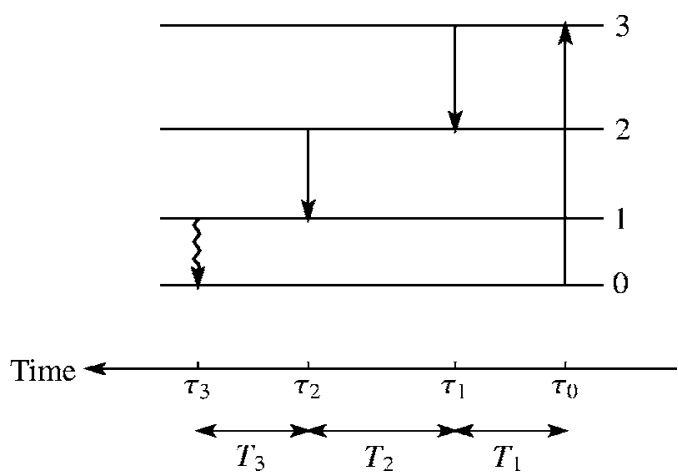

FIG. 12. The energy-level diagram corresponding to the double-sided Feynman diagram, Eq. (85).

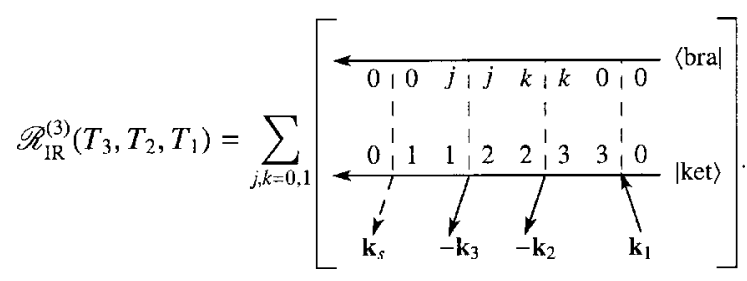

The 2D-IR signal discussed here is the real part of the twodimensional Fourier transform of $-\mathcal{R}_{\mathrm{IR}}^{(3)}\left(T_{3}, T_{2}, T_{1}\right)$ with respect to $T_{1}$ and $T_{2}$ :

$$
\begin{aligned}
S_{\text {rate }}\left(\Omega_{1}, \Omega_{2} ; T_{3}\right)= & \operatorname{Re}\left[-\int_{0}^{\infty} d T_{2} \int_{0}^{\infty} d T_{1} e^{i \Omega_{2} T_{2}+i \Omega_{1} T_{1}}\right. \\
& \left.\times \mathcal{R}_{\mathrm{IR}}^{(3)}\left(T_{3}, T_{2}, T_{1}\right)\right] .
\end{aligned}
$$

In a similar fashion to the preceding section, the volume of this $2 \mathrm{D}$ signal $S_{\text {rate }}\left(\Omega_{1}, \Omega_{2} ; T_{3}\right)$ is obtained as

$$
\begin{aligned}
V_{\text {rate }}\left(T_{3}\right)= & \int_{-\infty}^{\infty} d \Omega_{1} \int_{-\infty}^{\infty} d \Omega_{2} S_{\text {rate }}\left(\Omega_{1}, \Omega_{2} ; T_{3}\right) \\
= & \frac{(2 \pi)^{2} \mu_{0}^{4} Q_{10} Q_{21} Q_{32} Q_{30}}{\hbar^{3}} \\
& \times \operatorname{Im}\left[-\left\langle\left\langle 10\left|\hat{G}_{11}\left(T_{3}\right)\right| 10\right\rangle\right\rangle\right]\left(\rho_{0}^{\mathrm{eq}}\right)_{00} .
\end{aligned}
$$

Making a comparison between Eqs. (82) and (87), we obtain the following identity:

$$
k\left(T_{3}\right)=\frac{V_{\text {rate }}\left(T_{3}\right)}{\int_{T_{3}}^{\infty} d t V_{\text {rate }}(t)}=-\frac{d}{d T_{3}} \ln \left[\int_{T_{3}}^{\infty} d t V_{\text {rate }}(t)\right],
$$

which provides an exact connection between the reaction rate and the 2D-IR signal. Notice that as mentioned before, the definition of the reaction rate, Eq. (82) or Eq. (88), makes sense only for nonoscillatory motion of $\left\langle\left\langle 10\left|\hat{G}_{11}(t)\right| 10\right\rangle\right\rangle$ or $V_{\text {rate }}\left(T_{3}\right)$. In weak dissipation, $V_{\text {rate }}\left(T_{3}\right)$ oscillates between positive and negative values, and hence Eq. (88) diverges.

Here, let $\tau_{\text {mol }}$ be the time for transient behavior of $k(t)$ to relax. If the third delay time $T_{3}$ is greater than $\tau_{\text {mol }}$, Eq. (88) can be written as 


$$
k_{\mathrm{rxn}}=-\frac{d}{d T_{3}} \ln \left[\int_{T_{3}}^{\infty} d t V_{\text {rate }}(t)\right],
$$

and can be recast into a very simple form

$$
V_{\text {rate }}\left(T_{3}\right) \propto \exp \left(-k_{\mathrm{rxn}} T_{3}\right),
$$

that is to say, the volume of the 2D-IR signal $S_{\text {rate }}\left(\Omega_{1}, \Omega_{2} ; T_{3}\right)$ decays exponentially with the reaction rate constant $k_{\mathrm{rxn}}$ for the time region $T_{3}>\tau_{\mathrm{mol}}$. This formula is one of the main results presented in this paper. We can determine the rate constant for a nonadiabatic proton transfer reaction directly from $2 \mathrm{D}$-IR signals.

Figure 13 shows the three-dimensional plots of $S_{\text {rate }}\left(\Omega_{1}, \Omega_{2} ; T_{3}\right)$ calculated under the two-state-jump approximation together with their volumes as a function of $\Omega_{1}$ and $\Omega_{2}$ at the temperature $300 \mathrm{~K}$ for (a) $\omega_{10} T_{3}=0$, (b) $\omega_{10} T_{3}=0.2$, (c) $\omega_{10} T_{3}=10$, and (d) $\omega_{10} T_{3}=15$, where $1 / \omega_{10}$ $=1.66 \mathrm{ps}$ for $\omega_{10}=20 \mathrm{~cm}^{-1}$. The LL coupling strength, the SL coupling strength, and the correlation time of the bath noise are chosen to be

$$
\zeta_{\mathrm{LL}}=2.5 \omega_{10}, \quad \zeta_{\mathrm{SL}}{ }^{\prime}=0.5, \quad \tau_{c}=\frac{1}{\gamma}=\frac{1}{6 \omega_{10}},
$$

respectively. In addition, Fig. 14 shows the change of their volume $V_{\text {rate }}\left(T_{3}\right)$ as the function of time $T_{3}$. We see that $V_{\text {rate }}\left(T_{3}\right)$ decays exponentially after the transient region, $\omega_{10} T_{3}<\omega_{10} \tau_{\mathrm{mol}} \sim 2.5$. By using the formula, Eq. (90), and the volumes of panels (c) and (d) in Fig. 13 the reaction rate constant $k_{\mathrm{rxn}}$ can be evaluated as $0.113 \omega_{10}$, and this value accords well with the value in Fig. 11.

\section{(a) $\omega_{10} T_{3}=0$}

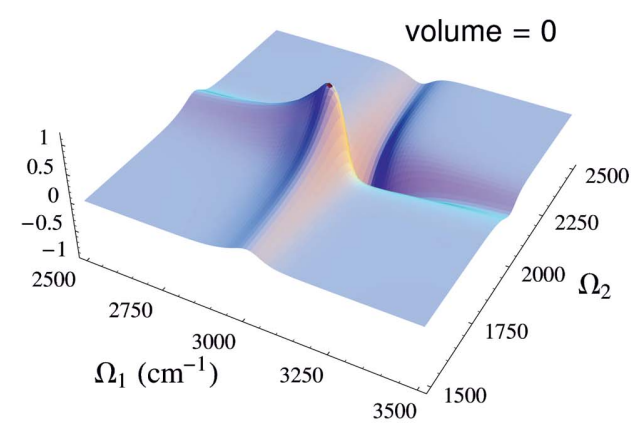

(c) $\omega_{10} T_{3}=10$

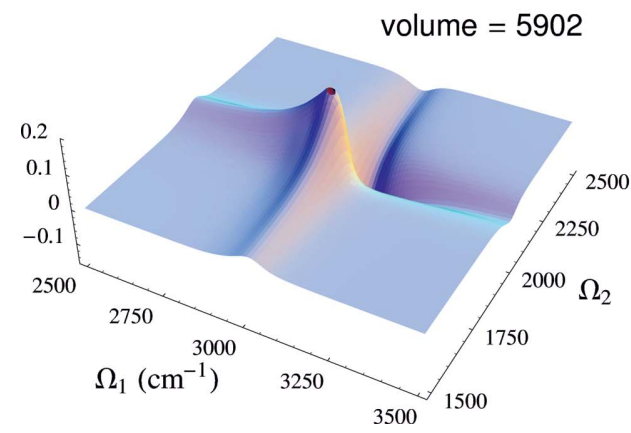

In Fig. 15, we show the rate constant $k_{\text {rxn }}$ calculated from Eq. (90) as a function of the LL coupling strength $\zeta_{\mathrm{LL}}$ with $\zeta_{\mathrm{SL}}{ }^{\prime}=0.2 \zeta_{\mathrm{LL}} / \omega_{10}$ for the parameters $\gamma=6 \omega_{10}$ and $\beta$ $=1 /\left(300 k_{\mathrm{B}}\right)$. We observe that in the strong coupling region the rate constant $k_{\text {rxn }}$ decreases with the increasing coupling strength $\zeta_{\mathrm{LL}}$ in the form $\sim \zeta_{\mathrm{LL}}{ }^{-1}$. The system-bath coupling destroys the coherence between the left and right well; the friction suppresses the transfer event via quantum tunneling [see also Eqs. (92) and (93)]. This behavior of $k_{\mathrm{rxn}}$ may correspond to the spatial-diffusion-controlled rate in the Kramers' theory. ${ }^{7,78}$ On the other hand, in the weak coupling region, we cannot evaluate the value of $k_{\mathrm{rxn}}$ because the volume $V_{\text {rate }}\left(T_{3}\right)$ oscillates between the positive and negative values as mentioned before. In this region, the chemical reaction rate constant cannot be defined due to the oscillation of quantum coherence, where the phenomenological rate law, Eqs. (C1) and (C2), cannot be applied.

We next consider the temperature dependence of the rate constant $k_{\text {rxn }}$ calculated from Eq. (90). Figure 16 depicts the plots of $k_{\mathrm{rxn}}$ as a function of inverse temperature $\beta$ within the high-temperature approximation $\beta \hbar \gamma \ll 1$ and the nonadiabatic limit condition $1 \ll \beta \hbar \omega_{\text {well }}$. The closed circle denotes the crossover inverse temperature $\beta_{c}$ from the incoherent region to the coherent region. As is evident from the plots, in the inverse temperature region below $\beta_{c}$, i.e., the incoherent region, the value of $k_{\mathrm{rxn}}$ decreases with increasing temperature. A similar phenomenon is discussed by Topaler and Makri. ${ }^{79}$ This is because the friction, which suppresses the tunneling, increases as the temperature increases. To illustrate this, we consider the case of the Gaussian-white noise

\section{(b) $\omega_{10} T_{3}=0.2$}

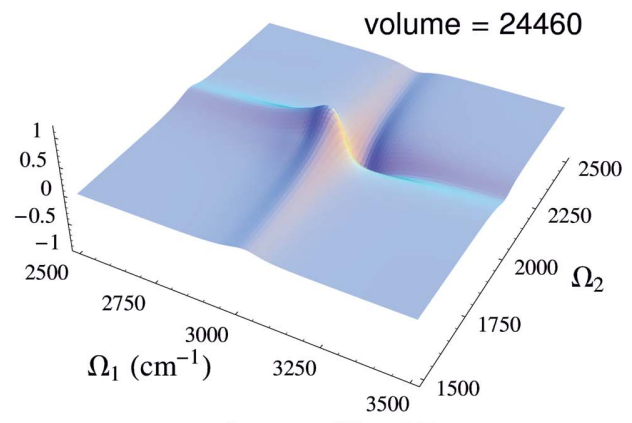

(d) $\omega_{10} T_{3}=15$

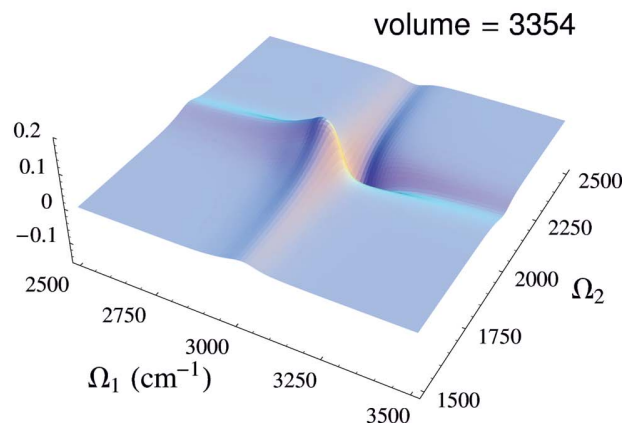

FIG. 13. (Color) Two-dimensional IR signal $S_{\text {rato }}\left(\Omega_{1}, \Omega_{2} ; T_{3}\right)$ calculated for the system in Fig. 1 under the two-state-jump approximation. The relevant transition frequencies are $\omega_{21}=2000 \mathrm{~cm}^{-1}, \omega_{30}=3000 \mathrm{~cm}^{-1}$, and $\omega_{10}=20 \mathrm{~cm}^{-1}\left(1 / \omega_{10}=1.66 \mathrm{ps}\right)$. The system-bath parameters are $\zeta_{L L}=2.5 \omega_{10}, \zeta_{S L}^{\prime}=0.5, \gamma$ $=6 \omega_{10}$, and $\beta=2.41 \times 10^{20}(300 \mathrm{~K})$. The normalization of each graph is such that the signal observed at $\left(\Omega_{1}, \Omega_{2}\right)=(3000,2000) \mathrm{cm}^{-1}$ in the panel $(\mathrm{a})$ is unity. 


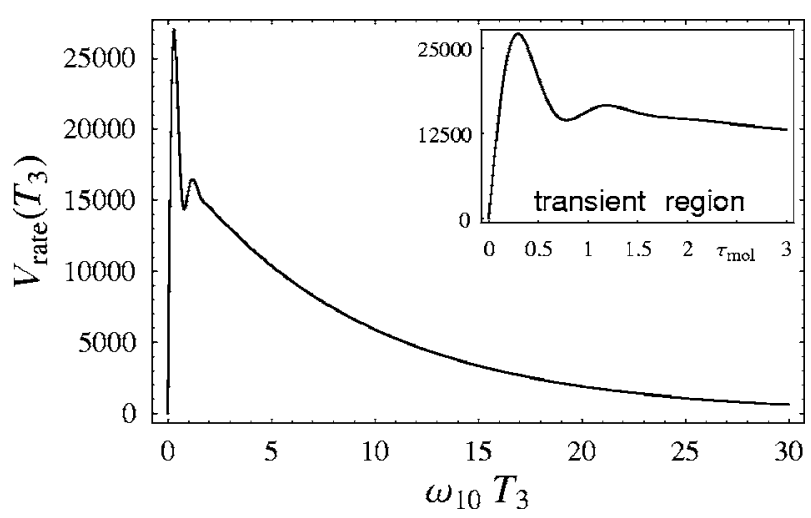

FIG. 14. The volume of the 2D signal in Fig. 13 is plotted as the function of the third delay time $T_{3}$, where $1 / \omega_{10}=1.66 \mathrm{ps}$ for $\omega_{10}=20 \mathrm{~cm}^{-1}$. After the transient region $T_{3}<\tau_{\text {mol }} \sim 2.5 / \omega_{10}$, the volume decays exponentially with the reaction rate constant $k_{\mathrm{rxn}}: V_{\text {rate }}\left(T_{3}\right) \propto \exp \left(-k_{\mathrm{rxn}} T_{3}\right)$ for $T_{3}>\tau_{\text {mol }}$.

bath in the absence of the SL coupling term, i.e., $K_{\mathrm{SL}}=0$, again. In this case, we can obtain the following simple expression for the rate constant $k_{\mathrm{rxn}}$ :

$$
k_{\mathrm{rxn}}^{\mathrm{W}}=\frac{\omega_{10}^{2}}{\zeta_{\mathrm{eff}}(\beta)+\sqrt{\zeta_{\mathrm{eff}}(\beta)^{2}-\omega_{10}^{2}}},
$$

where $\zeta_{\text {eff }}(\beta)$ is the effective coupling strength expressed as

$$
\zeta_{\text {eff }}(\beta)=\frac{2 M Q_{10}^{2}}{\hbar^{2}} \cdot \frac{\zeta_{\text {LL }}}{\beta} .
$$

Of course, Eq. (92) is valid under the following condition:

$$
\zeta_{\text {eff }}(\beta) \geqslant \omega_{10} \Leftrightarrow \beta \leqslant \frac{2 M Q_{10}^{2}}{\hbar^{2} \omega_{10}} \zeta_{L L} \equiv \beta_{c}^{\mathrm{W}},
$$

which determines the crossover point for the Gaussian-white noise bath case, $\beta_{c}^{\mathrm{W}}$. Equations (92) and (93) clearly show that the rise in temperature effectively intensifies the systembath coupling strength, and accordingly gives rise to the decrease of the rate.

As demonstrated above, two-dimensional IR spectroscopy can evaluate the rate constant of a nonadiabatic proton transfer reaction in the condensed phase. Viewed in this

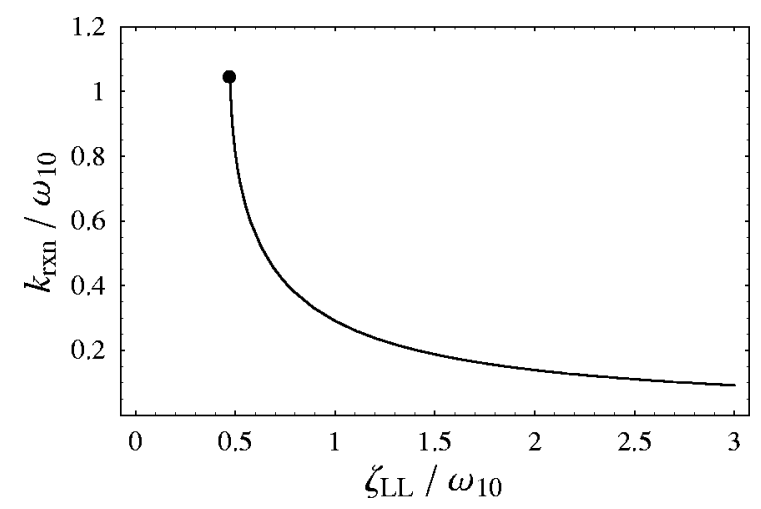

FIG. 15. The rate constant $k_{\text {rxn }}$ calculated from Eq. (90) is plotted as a function of the LL coupling strength $\zeta_{L L}$. The system-bath parameters are $\zeta_{S L}{ }^{\prime}=0.2 \zeta_{L L} / \omega_{10}, \gamma=6 \omega_{10}$, and $\beta=2.41 \times 10^{20}(300 \mathrm{~K})$. In the strong coupling region, the value of $k_{\mathrm{rxn}}$ decreases with $\zeta_{L L}$. In the weak coupling region below $\zeta_{L L}=0.47 \omega_{10}$, on the other hand, the rate constant $k_{\text {rxn }}$ cannot be defined due to quantum coherence oscillation. We denote the lower limit of $k_{\mathrm{rxn}}$ by the closed circle.

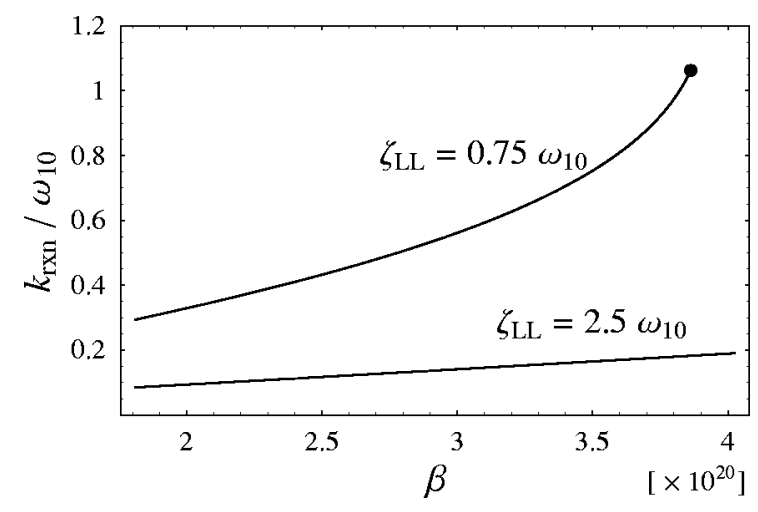

FIG. 16. The rate constant $k_{\text {rxn }}$ calculated from Eq. (90) are plotted as a function of $\beta$ for $\zeta_{L L}=0.75 \omega_{10}$ and $\zeta_{L L}=2.5 \omega_{10}$ within the high-temperature approximation and the nonadiabatic limit condition: $\beta \hbar \gamma \ll 1 \ll \beta \hbar \omega_{\text {well }}$. The other system-bath parameters are $\zeta_{S L}{ }^{\prime}=0.25 \zeta_{L L} / \omega_{10}$ and $\gamma=6 \omega_{10}$. The closed circle denotes the crossover point $\beta_{c}=1 /\left(k_{B} T_{c}\right)$ from the incoherent to the coherent phase. In the temperature region above $T_{c}$, the values of $k_{\text {rxn }}$ decrease with increasing temperature. In the temperature below $T_{c}$, on the other hand, the rate constant $k_{\mathrm{rxn}}$ cannot be defined.

light, multidimensional vibrational spectroscopy can be regarded as a novel technique for measuring chemical reaction rate constants. The evaluation of the rate constants by means of the multidimensional vibrational spectroscopy may inform us of phenomena which have been overlooked so far.

\section{CONCLUSION}

In this paper we investigated the nonadiabatic proton transfer reaction system in the condensed phase by means of 2D-IR spectroscopy. We considered a double-well potential system coupled to a colored noise bath with a linear-linear and square-linear system-bath interaction. We discuss the dynamics of the double-well system under infrared (IR) laser excitations from a Gaussian-Markovian quantum FokkerPlanck equation approach. 2D-IR signals were calculated within the two-state-jump approximation for various noise correlation time, system-bath coupling parameters, and temperatures. We investigated transition rates between the tunneling splitting levels as well as the reaction rates, which relates to the coherence between the splitting levels, with the use of the third-order optical processes. One of the important conclusions of this work was that the transition rates and the chemical reaction rates involved in the tunneling process were directly evaluated from the isolated peaks in the twodimensional frequency domain signals. This became possible because multidimensional spectroscopy can utilize phase matching conditions and present the signal in 2D frequency space to separate the contribution from different Liouville paths. We should emphasize that we discussed the 2D-IR signal, not only peak heights but also peak volumes, which allowed us to eliminate the contribution from the bathinduced coherence transfer process to the signal and hence the vibrational excitation and relaxation dynamics induced by the dissipative environment can be separated from all other processes. Moreover, the reaction rate constant can be directly evaluated; the 2D-IR spectroscopy can be regarded as a novel technique for measuring chemical reaction rate constants. 
The formalism presented in this paper provides a framework for the study of tunneling dynamics in a dissipative environment and may be extended to deeper potential well system which involves more than two tunneling splitting levels. In such case, the interplay between the thermal activation, dissipation, and tunneling processes become an interesting problem, and applications of multidimensional spectroscopy may provide in-depth understanding of chemical reaction dynamics in condensed phases. Here, we limited our study to vibrational spectroscopy of the proton transfer reaction, but we may apply a similar method for resonant spectroscopy to study a nonadiabatic electron transfer reaction.

In this paper we outlined a simple case to illustrate the complex issues associated with the numerous factors contributing to the tunneling process upon nonlinear spectroscopic signals. The simplicity of the model system, in particular, restricting the reaction coordinate to one dimension and as- suming the short laser pulses with very narrow bandwidth may limit the value of any direct comparisons to actual future experiments. However, for this paper the use of a simple system was very advantageous in clarifying the issues that must be considered in realistic and experimental cases. It is important to investigate more realistic system such as malonaldehyde by means of 2D-IR spectroscopy, which is sensitive to the characters of systems. ${ }^{80}$ The study for more realistic system to explore the possibility of actual experiments is left for future studies.

\section{ACKNOWLEDGMENTS}

The authors wish to express their gratitude to Dr. Oliver Kühn for fruitful discussions. Y.T. is thankful for the financial support from a Grant-in-Aid for Scientific Research (Grant No. A 15205005 from Japan Society for the Promotion of Science and Morino Science Foundation.

\section{APPENDIX A: IR RESPONSE FUNCTION FOR GAUSSIAN-MARKOVIAN NOISE BATH}

In this appendix, we derive the reduced response function of a quantum system coupled to a Gaussian-Markovian noise bath. The initial condition of the total system is assumed to be the factorized form as

$$
\hat{\rho}_{\mathrm{tot}}(t \rightarrow-\infty)=\hat{\rho}_{A}(-\infty) \otimes \hat{\rho}_{B}^{\mathrm{eq}},
$$

where $\hat{\rho}_{A}(-\infty)$ is the initial state of the system $A$ and $\hat{\rho}_{B}^{\text {eq }}$ is the thermal equilibrium state of the bath. However, Eq. (A1) is not the equilibrium state of the system $A+B$, because this neglects the correlated effects of the system-bath interaction. Here, note that the system comes to this correlated equilibrium state after sufficiently long time evolution, even though we started from the factorized initial condition. We may regard this correlated equilibrium state (at $t=t_{I}$ ) as the correlated initial condition of the total system. ${ }^{60}$ Then, the IR response function, Eq. (20), is expressed in the path integral form with the initial condition, Eq. (A1), as

$$
\begin{aligned}
& \int_{-\infty}^{\infty} d Q \int_{-\infty}^{\infty} d Q^{\prime} \delta\left(Q-Q^{\prime}\right) \int_{-\infty}^{\infty} d Q_{i} \int_{-\infty}^{\infty} d Q_{i}^{\prime} \int_{Q(-\infty)=Q_{i}}^{Q\left(t_{3}+t_{2}+t_{1}+t_{I}\right)=Q} \mathcal{D}[Q(t)] \int_{Q^{\prime}(-\infty)=Q_{i}^{\prime}}^{Q^{\prime}\left(t_{3}+t_{2}+t_{1}+t_{I}\right)=Q^{\prime}} \mathcal{D}\left[Q^{\prime}(t)\right] \mu\left(Q\left(t_{3}+t_{2}+t_{1}+t_{I}\right)\right) \\
& \quad \times \frac{i}{\hbar} \mathcal{X}\left(t_{2}+t_{1}+t_{I}\right) \frac{i}{\hbar} \mathcal{X}\left(t_{1}+t_{I}\right) \frac{i}{\hbar} \mathcal{X}\left(t_{I}\right) \exp \left(\frac{i}{\hbar} S_{A}[Q]\right) F_{\mathrm{FV}}\left[Q, Q^{\prime}\right] \exp \left(-\frac{i}{\hbar} S_{A}\left[Q^{\prime}\right]\right) \rho_{A}\left(Q_{i}, Q_{i}^{\prime} ;-\infty\right),
\end{aligned}
$$

with

$$
\mathcal{X}(t) \equiv \mu(Q(t))-\mu\left(Q^{\prime}(t)\right)
$$

where $S_{A}[Q]$ is the action defined by

$$
S_{A}[Q]=\int_{-\infty}^{t} d s\left[\frac{M}{2} \dot{Q}(s)^{2}-U(Q(s))\right],
$$

and $F_{\mathrm{Fv}}\left[Q, Q^{\prime}\right]$ is the Feynman-Vernon influence functional given by ${ }^{81-83}$

$$
\begin{aligned}
F_{\mathrm{FV}}\left[Q, Q^{\prime}\right]= & \exp \left(-\frac{1}{\hbar} \sum_{j} \frac{c_{j}^{2}}{2 m_{j} \omega_{j}} \int_{-\infty}^{t} d s_{1} \int_{-\infty}^{s_{1}} d s_{2}\left[V\left(Q\left(s_{1}\right)\right)-V\left(Q^{\prime}\left(s_{1}\right)\right)\right]\right. \\
& \times\left\{\left[V\left(Q\left(s_{2}\right)\right)-V\left(Q^{\prime}\left(s_{2}\right)\right)\right] \operatorname{coth}\left(\frac{\beta \hbar \omega_{j}}{2}\right) \cos \left(\omega_{j}\left(s_{1}-s_{2}\right)\right)-i\left[V\left(Q\left(s_{2}\right)\right)+V\left(Q^{\prime}\left(s_{2}\right)\right)\right] \sin \left(\omega_{j}\left(s_{1}-s_{2}\right)\right)\right\} \\
& \left.-\frac{i}{\hbar} \sum_{j} \frac{c_{j}^{2}}{2 m_{j} \omega_{j}^{2}} \int_{-\infty}^{t} d s\left[V(Q(s))^{2}-V\left(Q^{\prime}(s)\right)^{2}\right]\right) .
\end{aligned}
$$

Within the high-temperature approximation, $\operatorname{coth}(\beta \hbar \gamma) \simeq 1 /(\beta \hbar \gamma)$, we rewrite Eq. (A5) by using Eqs. (8), (10), and (11) as 


$$
F_{\mathrm{FV}}\left[Q, Q^{\prime}\right]=\exp \left(-\int_{-\infty}^{t} d s_{1} \hat{\Phi}\left(s_{1}\right) e^{-\gamma s_{1}}\left[-\int_{-\infty}^{s_{1}} d s_{2} e^{\gamma s_{2}} \hat{\Theta}\left(s_{2}\right)+C_{0}\right]\right),
$$

with

$$
\begin{aligned}
& \Phi(s) \equiv \frac{i}{\hbar}\left[V(Q(s))-V\left(Q^{\prime}(s)\right)\right], \\
& \Theta(s) \equiv \frac{i \zeta \gamma}{2}\left\{i\left[\frac{\partial V(Q(s))}{\partial Q(s)} M \dot{Q}(s)+\frac{\partial V\left(Q^{\prime}(s)\right)}{\partial Q^{\prime}(s)} M \dot{Q}^{\prime}(s)\right]+\frac{2 M}{\beta \hbar}\left[V(Q(s))-V\left(Q^{\prime}(s)\right)\right]\right\},
\end{aligned}
$$

and

$$
C_{0}=\frac{M}{2} \zeta \gamma\left[V\left(Q_{i}\right)+V\left(Q_{i}^{\prime}\right)\right]
$$

To calculate Eq. (A2) further, we introduce four auxiliary operators: ${ }^{84} \hat{\sigma}_{n}^{(3)}\left(t_{3}, t_{2}, t_{1}\right), \hat{\sigma}_{n}^{(2)}\left(t_{2}, t_{1}\right), \hat{\sigma}_{n}^{(1)}\left(t_{1}\right)$, and $\hat{\rho}_{n}^{\text {eq }}$.

First, we define $\hat{\sigma}_{n}^{(3)}\left(t_{3}, t_{2}, t_{1}\right)$ by its matrix element as

$$
\begin{aligned}
\sigma_{n}^{(3)}\left(Q, Q^{\prime} ; t_{3}, t_{2}, t_{1}\right)= & \int_{-\infty}^{\infty} d Q_{i} \int_{-\infty}^{\infty} d Q_{i}^{\prime} \int_{Q(-\infty)=Q_{i}}^{Q\left(t_{3}+t_{2}+t_{1}+t_{I}\right)=Q} \mathcal{D}[Q(t)] \int_{Q^{\prime}(-\infty)=Q_{i}^{\prime}}^{Q^{\prime\left(t_{3}+t_{2}+t_{1}+t_{I}\right)=Q^{\prime}}} \mathcal{D}\left[Q^{\prime}(t)\right] \\
& \times \frac{i}{\hbar} \mathcal{X}\left(t_{2}+t_{1}+t_{I}\right) \frac{i}{\hbar} \mathcal{X}\left(t_{1}+t_{I}\right) \frac{i}{\hbar} \mathcal{X}\left(t_{I}\right)\left\{e^{-\gamma\left(t_{3}+t_{2}+t_{1}+t_{I}\right)}\left[-\int_{-\infty}^{t_{3}+t_{2}+t_{1}+t_{I}} d s e^{\gamma s} \hat{\Theta}(s)+C_{0}\right]\right\}^{n} \\
& \times \exp \left(\frac{i}{\hbar} S_{A}[Q]\right) F_{\mathrm{FV}}\left[Q, Q^{\prime}\right] \exp \left(-\frac{i}{\hbar} S_{A}\left[Q^{\prime}\right]\right) \rho_{A}\left(Q_{i}, Q_{i}^{\prime} ;-\infty\right),
\end{aligned}
$$

for integer $n \geqslant 0$. Using Eq. (A10), the third-order IR response function is expressed as

$$
R_{\mathrm{IR}}^{(3)}\left(t_{3}, t_{2}, t_{1}\right)=\operatorname{Tr}_{A}\left\{\mu(\hat{Q}) \hat{\sigma}_{0}^{(3)}\left(t_{3}, t_{2}, t_{1}\right)\right\} .
$$

Differentiating $\sigma_{n}^{(3)}\left(q, q^{\prime} ; t_{3}, t_{2}, t_{1}\right)$ with respect to $t_{3}$, we obtain the following recurrence formula:

$$
\begin{aligned}
\frac{\partial}{\partial t_{3}} \sigma_{n}^{(3)}\left(Q, Q^{\prime} ; t_{3}, t_{2}, t_{1}\right) & \\
& =-\left[i \mathcal{L}_{A}\left(Q, Q^{\prime}\right)+n \gamma\right] \sigma_{n}^{(3)}\left(Q, Q^{\prime} ; t_{3}, t_{2}, t_{1}\right)-\Phi\left(Q, Q^{\prime}\right) \sigma_{n+1}^{(3)}\left(Q, Q^{\prime} ; t_{3}, t_{2}, t_{1}\right)-n \Theta\left(Q, Q^{\prime}\right) \sigma_{n-1}^{(3)}\left(Q, Q^{\prime} ; t_{3}, t_{2}, t_{1}\right),
\end{aligned}
$$

where

$$
i \mathcal{L}_{A}\left(Q, Q^{\prime}\right)=\frac{i}{\hbar}\left[-\frac{\hbar^{2}}{2 M}\left(\frac{\partial^{2}}{\partial Q^{2}}-\frac{\partial^{2}}{\partial Q^{\prime 2}}\right)+U(Q)-U\left(Q^{\prime}\right)\right]
$$

is the Liouvillian of the system $A$ in the coordinate representation. Relaxation operators $\Phi\left(Q, Q^{\prime}\right)$ and $\Theta\left(Q, Q^{\prime}\right)$ are obtained by changing $Q(t) \rightarrow Q, Q^{\prime}(t) \rightarrow Q^{\prime}, M \dot{Q}(s) \rightarrow(\hbar / i) \partial / \partial Q$, and $M \dot{Q}^{\prime}(s) \rightarrow-(\hbar / i) \partial / \partial Q^{\prime}$ in Eqs. (A7) and (A8), respectively.

In operator form, the recurrence formula, Eq. (A12), can be expressed as

$$
\frac{\partial}{\partial t_{3}} \hat{\sigma}_{n}^{(3)}\left(t_{3}, t_{2}, t_{1}\right)=-\left(i \hat{\mathcal{L}}_{A}+n \gamma\right) \hat{\sigma}_{n}^{(3)}\left(t_{3}, t_{2}, t_{1}\right)-\hat{\Phi} \hat{\sigma}_{n+1}^{(3)}\left(t_{3}, t_{2}, t_{1}\right)-n \hat{\Theta} \hat{\sigma}_{n-1}^{(3)}\left(t_{3}, t_{2}, t_{1}\right)
$$

where for any operand operator $\hat{f}$

$$
\begin{aligned}
& i \hat{\mathcal{L}}_{A} \hat{f}=\frac{i}{\hbar}\left[\frac{\hat{P}^{2}}{2 M}+U(\hat{Q})\right]^{\times} \hat{f}, \\
& \hat{\Phi} \hat{f}=\frac{i}{\hbar} V(\hat{Q})^{\times} \hat{f} \\
& \hat{\Theta} \hat{f}=\frac{i \zeta \gamma}{2}\left[i\left(\frac{\partial V(\hat{Q})}{\partial \hat{Q}} \hat{P} \hat{f}+\hat{f} \hat{P} \frac{\partial V(\hat{Q})}{\partial \hat{Q}}\right)+\frac{2 M}{\beta \hbar} V(\hat{Q})^{\times} \hat{f}\right] .
\end{aligned}
$$

The Laplace transform of Eq. (A14) with respect to $t_{3}$ can be expressed in the following matrix form as 


$$
\left[\begin{array}{c}
\hat{\sigma}_{0}^{(3)}\left[s_{3}, t_{2}, t_{1}\right) \\
\hat{\sigma}_{1}^{(3)}\left[s_{3}, t_{2}, t_{1}\right) \\
\hat{\sigma}_{2}^{(3)}\left[s_{3}, t_{2}, t_{1}\right) \\
\vdots
\end{array}\right]=\hat{G}\left[s_{3}\right]\left[\begin{array}{c}
\hat{\sigma}_{0}^{(3)}\left(0, t_{2}, t_{1}\right) \\
\hat{\sigma}_{1}^{(3)}\left(0, t_{2}, t_{1}\right) \\
\hat{\sigma}_{2}^{(3)}\left(0, t_{2}, t_{1}\right) \\
\vdots
\end{array}\right],
$$

where $\hat{G}[s]$ is the tridiagonal matrix defined by

$$
\hat{G}[s]=\left[\begin{array}{ccccc}
s+i \hat{\mathcal{L}}_{A} & \hat{\Phi} & 0 & 0 & \ldots \\
\hat{\Theta} & s+\gamma+i \hat{\mathcal{L}}_{A} & \hat{\Phi} & 0 & \ldots \\
0 & 2 \hat{\Theta} & s+2 \gamma+i \hat{\mathcal{L}}_{A} & \hat{\Phi} & \ddots \\
0 & 0 & 3 \hat{\Theta} & \ddots & \ddots \\
\vdots & \vdots & \ddots & \ddots & \ddots
\end{array}\right]^{-1}
$$

Second, we define the auxiliary operator $\hat{\sigma}_{n}^{(2)}\left(t_{2}, t_{1}\right)$ by its matrix element as

$$
\begin{aligned}
\sigma_{n}^{(2)}\left(Q, Q^{\prime} ; t_{2}, t_{1}\right)= & \int_{-\infty}^{\infty} d Q_{i} \int_{-\infty}^{\infty} d Q_{i}^{\prime} \int_{Q(-\infty)=Q_{i}}^{Q\left(t_{2}+t_{1}+t_{I}\right)=Q} \mathcal{D}[Q(t)] \int_{Q^{\prime}(-\infty)=Q_{i}^{\prime}}^{Q^{\prime}\left(t_{2}+t_{1}+t_{I}\right)=Q^{\prime}} \mathcal{D}\left[Q^{\prime}(t)\right] \\
& \times \frac{i}{\hbar} \mathcal{X}\left(t_{1}+t_{I}\right) \frac{i}{\hbar} \mathcal{X}\left(t_{I}\right)\left\{e^{-\gamma\left(t_{2}+t_{1}+t_{I}\right)}\left[-\int_{-\infty}^{t_{2}+t_{1}+t_{I}} d s e^{\gamma s} \Theta(s)+C_{0}\right]\right\}^{n} \\
& \times \exp \left(\frac{i}{\hbar} S_{A}[Q]\right) F_{\mathrm{FV}}\left[Q, Q^{\prime}\right] \exp \left(-\frac{i}{\hbar} S_{A}\left[Q^{\prime}\right]\right) \rho_{A}\left(Q_{i}, Q_{i}^{\prime} ;-\infty\right),
\end{aligned}
$$

for integer $n \geqslant 0$, where the first auxiliary operator $\hat{\sigma}_{n}^{(3)}\left(0, t_{2}, t_{1}\right)$ is given by

$$
\hat{\sigma}_{n}^{(3)}\left(0, t_{2}, t_{1}\right)=\frac{i}{\hbar} \mu(\hat{Q})^{\times} \hat{\sigma}_{n}^{(2)}\left(t_{2}, t_{1}\right) .
$$

The similar procedure to Eqs. (A12)-(A18) allows us to write

$$
\left[\begin{array}{c}
\hat{\sigma}_{0}^{(3)}\left[0, s_{2}, t_{1}\right) \\
\hat{\sigma}_{1}^{(3)}\left[0, s_{2}, t_{1}\right) \\
\hat{\sigma}_{2}^{(3)}\left[0, s_{2}, t_{1}\right) \\
\vdots
\end{array}\right]=\frac{i}{\hbar} \mu(\hat{Q})^{\times} \hat{G}\left[s_{2}\right]\left[\begin{array}{c}
\hat{\sigma}_{0}^{(2)}\left(0, t_{1}\right) \\
\hat{\sigma}_{1}^{(2)}\left(0, t_{1}\right) \\
\hat{\sigma}_{2}^{(2)}\left(0, t_{1}\right) \\
\vdots
\end{array}\right] .
$$

Finally, we define the auxiliary operator $\hat{\sigma}_{n}^{(1)}\left(t_{1}\right)$ by its matrix element as

$$
\begin{aligned}
\sigma_{n}^{(1)}\left(Q, Q^{\prime} ; t_{1}\right)= & \int_{-\infty}^{\infty} d Q_{i} \int_{-\infty}^{\infty} d Q_{i}^{\prime} \int_{Q(-\infty)=Q_{i}}^{Q\left(t_{1}+t_{I}\right)=Q} \mathcal{D}[Q(t)] \int_{Q^{\prime}(-\infty)=Q_{i}^{\prime}}^{Q^{\prime}\left(t_{1}+t_{I}\right)=Q^{\prime}} \mathcal{D}\left[Q^{\prime}(t)\right] \frac{i}{\hbar} \mathcal{X}\left(t_{I}\right)\left\{e^{-\gamma\left(t_{1}+t_{I}\right)}\left[-\int_{-\infty}^{t_{1}+t_{I}} d s e^{\gamma s} \Theta(s)+C_{0}\right]\right\}^{n} \\
& \times \exp \left(\frac{i}{\hbar} S_{A}[Q]\right) F_{\mathrm{FV}}\left[Q, Q^{\prime}\right] \exp \left(-\frac{i}{\hbar} S_{A}\left[Q^{\prime}\right]\right) \rho_{A}\left(Q_{i}, Q_{i}^{\prime} ;-\infty\right)
\end{aligned}
$$

with

$$
\sigma_{n}^{(1)}(0)=\frac{i}{\hbar} \mu(\hat{Q})^{\times} \hat{\rho}_{n}^{\mathrm{eq}}
$$

for integer $n \geqslant 0$, where the second auxiliary operator $\hat{\sigma}_{n}^{(2)}\left(0, t_{1}\right)$ is given by

$$
\hat{\sigma}_{n}^{(2)}\left(0, t_{1}\right)=\frac{i}{\hbar} \mu(\hat{Q})^{\times} \hat{\sigma}_{n}^{(1)}\left(t_{1}\right) .
$$

We follow the procedure applied for Eqs. (A12)-(A22) and get 


$$
\left[\begin{array}{c}
\hat{\sigma}_{0}^{(2)}\left[0, s_{1}\right] \\
\hat{\sigma}_{1}^{(2)}\left[0, s_{1}\right] \\
\hat{\sigma}_{2}^{(2)}\left[0, s_{1}\right] \\
\vdots
\end{array}\right]=\frac{i}{\hbar} \mu(\hat{Q})^{\times} \hat{G}\left[s_{1}\right] \frac{i}{\hbar} \mu(\hat{Q})^{\times}\left[\begin{array}{c}
\hat{\rho}_{0}^{\mathrm{eq}} \\
\hat{\rho}_{1}^{\mathrm{eq}} \\
\hat{\rho}_{2}^{\mathrm{eq}} \\
\vdots
\end{array}\right] .
$$

Using Eqs. (A11), (A18), (A22), and (A26), we obtain the reduced description for the triple Laplace transform of the third-order IR response function by

$$
R_{\mathrm{IR}}^{(3)}\left[s_{3}, s_{2}, s_{1}\right]=\sum_{J=1}^{\infty} \sum_{K=1}^{\infty} \sum_{L=1}^{\infty} \operatorname{Tr}_{A}\left\{\mu(\hat{Q}) \hat{G}_{1 J}\left[s_{3}\right] \frac{i}{\hbar} \mu(\hat{Q})^{\times} \hat{G}_{J K}\left[s_{2}\right] \frac{i}{\hbar} \mu(\hat{Q})^{\times} \hat{G}_{K L}\left[s_{1}\right] \frac{i}{\hbar} \mu(\hat{Q})^{\times} \hat{\rho}_{L-1}^{\mathrm{eq}}\right\},
$$

where $\hat{G}_{J K}[s]$ is the element of the matrix $\hat{G}[s]$ in the $J$ th row and the $K$ th column (see Appendix B), and $\hat{\rho}_{L}^{\text {eq }}$ is the density operator of the correlated equilibrium state expressed as

$$
\hat{\rho}_{L-1}^{\mathrm{eq}}=\lim _{s \rightarrow 0} s \hat{G}_{L 1}[s] \hat{\rho}_{A}(-\infty) .
$$

\section{APPENDIX B: EVALUATION OF $G_{j k}[s]$}

To calculate Eq. (A27), we have to evaluate the elements of the matrix Eq. (A19). For this purpose, we generally consider an inverse matrix of the following tridiagonal matrix:

$$
\mathrm{D}_{0}=\left[\begin{array}{cccc}
a_{0} & b_{0} & 0 & \ldots \\
c_{0} & a_{1} & b_{1} & \ldots \\
0 & c_{1} & a_{2} & \ldots \\
\vdots & \vdots & \vdots & \ddots
\end{array}\right]
$$

which can be expressed in the recurrence formula,

$$
\mathrm{D}_{n}=\left[\begin{array}{c:c}
a_{n} & \mathrm{~B}_{n} \\
\hdashline \mathrm{C}_{n} & \mathrm{D}_{n+1}
\end{array}\right] \quad(n=0,1,2, \ldots),
$$

with

$$
\mathrm{B}_{n}=\left[\begin{array}{llll}
b_{n} & 0 & 0 & \ldots
\end{array}\right], \quad \mathrm{C}_{n}=\left[\begin{array}{llll}
c_{n} & 0 & 0 & \ldots
\end{array}\right]^{T} .
$$

The inverse matrix of Eq. (B2) is given by ${ }^{68}$

$$
\frac{1}{\mathrm{D}_{n}}=\left[\begin{array}{c:c}
z_{n} & -z_{n} \mathrm{~B}_{n} \frac{1}{\mathrm{D}_{n+1}} \\
\hdashline-\frac{1}{\mathrm{D}_{n+1}} \mathrm{C}_{n} z_{n} & \frac{1}{\mathrm{D}_{n+1}} \mathrm{C}_{n} z_{n} \mathrm{~B}_{n} \frac{1}{\mathrm{D}_{n+1}}+\frac{1}{\mathrm{D}_{n+1}}
\end{array}\right],
$$

with

$$
z_{n}=\frac{1}{a_{n}-\mathrm{B}_{n} \frac{1}{\mathrm{D}_{n+1}} \mathrm{C}_{n}},
$$

where fractional expressions mean the corresponding inverse matrices. Hence, we have

$$
\left(\frac{1}{\mathrm{D}_{n}}\right)_{11}=z_{n}=\frac{1}{a_{n}-b_{n} z_{n+1} c_{n}},
$$

$$
\begin{aligned}
& \left(\frac{1}{\mathrm{D}_{n}}\right)_{J 1}=\left(\frac{1}{\mathrm{D}_{n+1}}\right)_{J-1,1}\left(-c_{n}\right) z_{n}, \\
& \left(\frac{1}{\mathrm{D}_{n}}\right)_{1 K}=z_{n}\left(-b_{n}\right)\left(\frac{1}{\mathrm{D}_{n+1}}\right)_{1, K-1},
\end{aligned}
$$

and

$$
\begin{aligned}
\left(\frac{1}{\mathrm{D}_{n}}\right)_{J K}= & \left(\frac{1}{\mathrm{D}_{n+1}}\right)_{J-1,1} c_{n} z_{n} b_{n}\left(\frac{1}{\mathrm{D}_{n+1}}\right)_{1, K-1} \\
& +\left(\frac{1}{\mathrm{D}_{n+1}}\right)_{J-1, K-1},
\end{aligned}
$$

where $J \geqslant 2$ and $K \geqslant 2$.

By successive applications of Eqs. (B6)-(B9) to the elements of $\mathrm{D}_{0}^{-1}$ and setting the following condition for $N$ $<L$ :

$$
\prod_{\alpha=L}^{N}\left[z_{J+L-\alpha-1}\left(-c_{J+L-\alpha-2}\right)\right]=\prod_{\beta=L}^{N}\left[\left(-b_{\beta-1}\right) z_{\beta}\right]=1,
$$

we obtain

$$
\begin{aligned}
\left(\frac{1}{\mathrm{D}_{0}}\right)_{J K}= & \sum_{L=1}^{\min (J, K)}\left\{\prod_{\alpha=L}^{J-1}\left[z_{J+L-\alpha-1}\left(-c_{J+L-\alpha-2}\right)\right]\right. \\
& \left.\cdot z_{L-1} \cdot \prod_{\beta=L}^{K-1}\left[\left(-b_{\beta-1}\right) z_{\beta}\right]\right\},
\end{aligned}
$$

with

$$
z_{K}=\frac{1}{a_{K}-b_{K} \frac{1}{a_{K+1}-b_{K+1} \frac{1}{a_{K+2}-\cdots} c_{K+1}} c_{K}} .
$$

Finally, by putting $z_{N}=\hat{Z}_{N}[s], a_{N}=s+i \hat{\mathcal{L}}_{A}+N \gamma, b_{N}=\hat{\Phi}$, and $c_{N}=(N+1) \hat{\Theta}$, we obtain the elements of the matrix (A19) as 


$$
\hat{G}_{J K}[s]=\sum_{L=1}^{\min (J, K)}\left\{\frac{(J-1) !}{(L-1) !} \prod_{\alpha=L}^{J-1}\left\{\hat{Z}_{J-\alpha+L-1}[s](-\hat{\Theta})\right\} \cdot \hat{Z}_{L-1}[s] \cdot \prod_{\beta=L}^{K-1}\left\{(-\hat{\Phi}) \hat{Z}_{\beta}[s]\right\},\right.
$$

with

$$
\hat{Z}_{N}[s]=\frac{1}{s+i \hat{\mathcal{L}}_{A}+N \gamma-\hat{\Phi} \frac{N+1}{s+i \hat{\mathcal{L}}_{A}+(N+1) \gamma-\hat{\Phi} \frac{N+2}{s+i \hat{\mathcal{L}}_{A}+(N+2) \gamma-\cdots}} \hat{\Theta}},
$$

where we have set the condition for $N<L$,

$$
\prod_{\alpha=L}^{N}\left\{\hat{Z}_{j-\alpha+L-1}[s](-\hat{\Theta})\right\}=\prod_{\beta=L}^{N}\left\{(-\hat{\Phi}) \hat{Z}_{\beta}[s]\right\}=1 .
$$

\section{APPENDIX C: TWO-BODY CORRELATION FUNCTION FOR REACTION RATE}

Let $N_{L}(t)$ and $N_{R}(t)$ denote the population of the left $(L)$ and right $(R)$ wells, respectively (see Fig. 10). Then, for the present closed system, where $N_{L}(t)+N_{R}(t)$ is a constant, $N_{L}(t)$ and $N_{R}(t)$ obey the phenomenological rate equations,

$$
\begin{aligned}
& \frac{d}{d t} N_{L}(t)=-k_{R \leftarrow L} N_{L}(t)+k_{L \leftarrow R} N_{R}(t), \\
& \frac{d}{d t} N_{R}(t)=k_{R \leftarrow L} N_{L}(t)-k_{L \leftarrow R} N_{R}(t),
\end{aligned}
$$

$k_{R \leftarrow L}$ and $k_{L \leftarrow R}$ being specific rate constants in $L \rightarrow R$ and $R \rightarrow L$ directions. The deviation of $N_{R}(t)$ away from its equilibrium value $N_{R}^{\mathrm{eq}}$ therefore satisfies a simple linear relaxation law

$$
\frac{d}{d t} \delta N(t)=-k_{\mathrm{rXn}} \delta N(t),
$$

with $\delta N(t) \equiv N_{R}(t)-N_{R}^{\mathrm{eq}}$ and $k_{\mathrm{rxn}} \equiv k_{L \leftarrow R}+k_{R \leftarrow L}$; the solution to the equation is

$$
\delta N(t)=\delta N(0) \exp \left(-k_{\mathrm{rxn}} t\right)
$$

Since we have the relation $k_{R \leftarrow L} / k_{L \leftarrow R}=N_{R}^{\mathrm{eq}} / N_{L}^{\mathrm{eq}}=1$ for the present system, the determination of $k_{\mathrm{rxn}}$ is sufficient to find the rate constants. For simplicity of terminology, hence, we call $k_{\mathrm{rxn}}$ the rate constant. ${ }^{85}$ The equality, Eq. (C4), is, however, not correct for all times. For short time, we expect transient behavior that should not correspond to the exponential macroscopic decay; the phenomenological rate laws can only be right on a time scale that does not resolve the short time transient relaxation ${ }^{8,60,86,87}$ (see Fig. 11). The value of the rate constant $k_{\mathrm{rxn}}$ should, therefore, be determined by the asymptotic limit of $k(t)$ defined as

$$
k(t) \equiv-\frac{(d / d t) \delta N(t)}{\delta N(t)} .
$$

More noteworthy is that if $k(t)$ does not exhibit a plateau, then the phenomenological rate law in which $k_{L \leftarrow R}$ and $k_{R \leftarrow L}$ are constants is an invalid description of the chemical kinetics.

To apply Eq. (C5), all that is necessary is to perturb the population from the equilibrium state. ${ }^{88}$ If this perturbation is small enough, $(d / d t) \delta N(t)$ and $\delta N(t)$ are expected to be proportional to the strength of the perturbation. Here, note that Eq. (C3) does not depend on the form of perturbation, and this is guaranteed by the fluctuation-dissipation theorem. The theorist's linear response experiment uses a different nonthermal way to perturb the populations. Unconstrained by the experimental setup, theorists have at their disposal a whole slew of ways of perturbing a system. Hence, we introduce the perturbation for the right well written as

$$
U(Q) \rightarrow U(Q)-\epsilon \theta(Q),
$$

where $\theta(Q)$ is the Heaviside's step function and $\epsilon$ is a small positive constant. By turning on the perturbation for a sufficient time, the system comes to a perturbed equilibrium state, where the population of the left well is shifted to the right well compared with that in the unperturbed equilibrium state. After the system comes to the perturbed equilibrium state, the perturbation is turned off (this defines time $t=0$ ). Now the population, equilibrated with the perturbation, is no longer in equilibrium and will have to re-equilibrate to that of the unperturbed system. The change of the population in the right well $\delta N(t)$ is now defined by using the density operator as follows:

$$
\delta N(t)=\operatorname{Tr}\left\{\hat{\rho}_{\mathrm{tot}}(t) \theta(\hat{Q})\right\}-\operatorname{Tr}\left\{\hat{\rho}_{\mathrm{tot}}^{\mathrm{eq}} \theta(\hat{Q})\right\},
$$

where $^{77}$

$$
\begin{aligned}
\hat{\rho}_{\mathrm{tot}}(t)= & \hat{\rho}_{\mathrm{tot}}^{\mathrm{eq}}+\int_{-\infty}^{t} d t^{\prime} e^{-i \hat{H}_{\mathrm{tot}}\left(t-t^{\prime}\right) / \hbar} \\
& \times \frac{1}{i \hbar}\left[\hat{H}^{\mathrm{ext}}\left(t^{\prime}\right), \hat{\rho}_{\mathrm{tot}}^{\mathrm{eq}}\right] e^{-i \hat{H}_{\mathrm{tot}}\left(t-t^{\prime}\right) / \hbar},
\end{aligned}
$$

with $\hat{H}^{\text {ext }}(t)=-\epsilon \theta(\hat{Q}) \theta(-t)$. We can, therefore, obtain $k(t)$ as 


$$
\left.k(t)=\frac{\operatorname{Tr}\left\{\delta \hat{\theta}_{R} e^{-i \hat{\mathcal{L}}_{\mathrm{tot}} \frac{i}{\hbar}} \delta \hat{\theta}_{R}^{\times} \hat{\rho}_{\mathrm{tot}}^{\mathrm{eq}}\right\}}{\int_{t}^{\infty} d s \operatorname{Tr}\left\{\delta \hat{\theta}_{R} e^{-i \hat{\mathcal{L}}_{\mathrm{tot}} \mathrm{s}} \frac{i}{\hbar} \delta \hat{\theta}_{R} \times \hat{\rho}_{\mathrm{tot}}^{\mathrm{eq}}\right.}\right\},
$$

where we have set $\hat{\theta}_{R} \equiv \theta(\hat{Q})$ and $\delta \hat{\theta}_{R} \equiv \hat{\theta}_{R}-\left\langle\hat{\theta}_{R}\right\rangle$. Moreover, by using the Kubo identity for any operator $\hat{\mathcal{O}}{ }^{77}$

$$
\frac{i}{\hbar}\left[e^{\left.-\beta \hat{H}_{\mathrm{tot}}, \hat{\mathcal{O}}\right]}=e^{-\beta \hat{H}_{\mathrm{tot}}} \int_{0}^{\beta} d \lambda e^{\lambda \hat{H}_{\mathrm{tot}}} \frac{i}{\hbar}\left[\hat{\mathcal{O}}, \hat{H}_{\mathrm{tot}}\right] e^{-\lambda \hat{H}_{\mathrm{tot}}}\right.
$$

we can recast Eq. (C9) into

$$
k(t)=\frac{\left\langle\delta \dot{\hat{\theta}}_{R} ; \delta \hat{\theta}_{R}(t)\right\rangle}{\left\langle\delta \hat{\theta}_{R} ; \delta \hat{\theta}_{R}(t)\right\rangle},
$$

where $\langle\cdots ; \cdots\rangle$ is the canonical correlation defined by Eq. (79) and $\delta \dot{\theta}_{R}=\left[\delta \hat{\theta}, \hat{H}_{\text {tot }}\right] / i \hbar$. The reaction rates based on the linear response calculation was given by Yamamoto. ${ }^{4} \mathrm{~A}$ variety of nearly equivalent two-body correlation expressions were also introduced by other researchers. ${ }^{5,6,89}$

${ }^{1}$ Quantum Tunneling in Condensed Media, Modern Problems in Condensed Matter Sciences Vol. 34, edited by Y. Kagan and A. J. Leggett (North-Holland, Amsterdam, 1992).

${ }^{2}$ V. I. Gol'danskii, L. I. Trakhtenberg, and V. N. Fleurov, Tunneling Phenomena in Chemical Physics (Gordon and Breach, New York, 1989).

${ }^{3}$ D. DeVault, Quantum-Mechanical Tunneling in Biological Systems (Cambridge University Press, Cambridge, 1984).

${ }^{4}$ T. Yamamoto, J. Chem. Phys. 33, 281 (1960).

${ }^{5}$ D. Chandler, J. Chem. Phys. 68, 2959 (1978).

${ }^{6}$ W. H. Miller, S. D. Schwartz, and J. W. Tromp, J. Chem. Phys. 79, 4889 (1983).

${ }^{7}$ P. Hänggi, P. Talkner, and M. Borkovec, Rev. Mod. Phys. 62, 251 (1990).

${ }^{8}$ Y. Tanimura and P. G. Wolynes, J. Chem. Phys. 96, 8485 (1992).

${ }^{9}$ R. L. Redington, T. E. Redington, and J. M. Montgomery, J. Chem. Phys. 113, 2304 (2000).

${ }^{10}$ F. Madeja and M. Havenith, J. Chem. Phys. 117, 7162 (2002).

${ }^{11}$ H. Sekiya, Y. Nagashima, and Y. Nishimura, J. Chem. Phys. 92, 5761 (1990)

${ }^{12}$ K. Tanaka, H. Honjo, H. Kohguchi, Y. Ohshima, and Y. Endo, J. Chem. Phys. 110, 1969 (1999).

${ }^{13}$ R. G. Gordon, Adv. Magn. Reson. 3, 1 (1968)

${ }^{14}$ G. C. Schatz and M. A. Ratner, Quantum Mechanics in Chemistry (Prentice Hall, New Jersey, 1993).

${ }^{15}$ S. Mukamel, Principles of Nonlinear Optical Spectroscopy (Oxford University Press, New York, 1995).

${ }^{16}$ Y. Tanimura and S. Mukamel, J. Chem. Phys. 99, 9496 (1993).

${ }^{17}$ P. Hamm, M. Lim, and R. M. Hochstrasser, J. Phys. Chem. B 102, 6123 (1998).

${ }^{18}$ K. Okumura and Y. Tanimura, J. Chem. Phys. 106, 1687 (1997).

${ }^{19}$ K. Okumura and Y. Tanimura, J. Chem. Phys. 107, 2267 (1997).

${ }^{20}$ Y. Tanimura, Chem. Phys. 233, 217 (1998).

${ }^{21}$ A. Tokmakoff, M. J. Lang, D. S. Larsen, G. R. Fleming, V. Chernyak, and S. Mukamel, Phys. Rev. Lett. 79, 2702 (1997).

${ }^{22}$ K. Okumura and Y. Tanimura, Chem. Phys. Lett. 278, 175 (1997).

${ }^{23}$ M. Cho, K. Okumura, and Y. Tanimura, J. Chem. Phys. 108, 1326 (1998).

${ }^{24}$ K. Okumura, D. M. Jonas, and Y. Tanimura, Chem. Phys. 266, 237 (2001).

${ }^{25}$ N.-H. Ge, M. T. Zanni, and R. M. Hochstrasser, J. Phys. Chem. A 106, 962 (2002).

${ }^{26}$ R. Venkatramani and S. Mukamel, J. Chem. Phys. 117, 11089 (2002).

${ }^{27}$ T. Steffen and Y. Tanimura, J. Phys. Soc. Jpn. 69, 3115 (2000).

${ }^{28}$ Y. Tanimura and T. Steffen, J. Phys. Soc. Jpn. 69, 4095 (2000).
${ }^{29}$ T. Kato and Y. Tanimura, J. Chem. Phys. 117, 6221 (2002).

${ }^{30}$ T. Kato and Y. Tanimura, J. Chem. Phys. 120, 260 (2004).

${ }^{31}$ K. F. Everitt and J. L. Skinner, Chem. Phys. 266, 197 (2001).

${ }^{32}$ K. F. Everitt, E. Geva, and J. L. Skinner, J. Chem. Phys. 114, 1326 (2001).

${ }^{33}$ O. Kühn and Y. Tanimura, J. Chem. Phys. 119, 2155 (2003).

${ }^{34}$ K. Okumura, A. Tokmakoff, and Y. Tanimura, J. Chem. Phys. 111, 492 (1999).

${ }^{35}$ W. Zhao and J. C. Wright, Phys. Rev. Lett. 84, 1411 (2000).

${ }^{36}$ M. C. Asplund, M. T. Zanni, and R. M. Hochstrasser, Proc. Natl. Acad. Sci. U.S.A. 97, 8219 (2000).

${ }^{37}$ C. Scheurer, A. Piryatinski, and S. Mukamel, J. Am. Chem. Soc. 123, 3114 (2001).

${ }^{38}$ C. Scheurer and S. Mukamel, J. Chem. Phys. 116, 6803 (2002).

${ }^{39}$ K. A. Merchant, W. G. Noid, D. E. Thompson, R. Akiyama, R. F. Loring, and M. D. Fayer, J. Phys. Chem. B 107, 4 (2003).

${ }^{40}$ K. J. Kubarych, C. J. Milne, S. Lin, V. Astinov, and R. J. D. Miller, J. Chem. Phys. 116, 2016 (2002).

${ }^{41}$ O. Golonzka, N. Demirdöven, M. Khalil, and A. Tokmakoff, J. Chem. Phys. 113, 9893 (2000).

${ }^{42}$ L. J. Kaufman, J. Y. Heo, L. D. Ziegler, and G. R. Fleming, Phys. Rev. Lett. 88, 207402 (2002).

${ }^{43} \mathrm{~K}$. Tominaga and K. Yoshihara, J. Chin. Chem. Soc. (Taipei) 47, 631 (2000).

${ }^{44}$ J. C. Kirkwood and A. C. Albrecht, J. Raman Spectrosc. 31, 107 (2000).

${ }^{45}$ M. Cho, D. A. Blank, J. Sung, K. Park, S. Hahn, and G. R. Fleming, J. Chem. Phys. 112, 2082 (2000).

${ }^{46}$ K. Tominaga and K. Yoshihara, Phys. Rev. Lett. 74, 3061 (1995).

${ }^{47}$ T. Steffen and K. Duppen, Phys. Rev. Lett. 76, 1224 (1996).

${ }^{48}$ A. Tokmakoff and G. R. Fleming, J. Chem. Phys. 106, 2569 (1997).

${ }^{49}$ S. Woutersen and P. Hamm, J. Phys. Chem. B 104, 11316 (2000).

${ }^{50}$ S. Woutersen and P. Hamm, J. Chem. Phys. 115, 7737 (2001).

${ }^{51}$ M. T. Zanni, S. Gnanakaran, J. Stenger, and R. M. Hochstrasser, J. Phys. Chem. B 105, 6520 (2001).

${ }^{52}$ I. V. Rubtsov and R. M. Hochstrasser, J. Phys. Chem. B 106, 9165 (2002).

${ }^{53}$ A. Tokmakoff and M. D. Fayer, J. Chem. Phys. 103, 2810 (1995).

${ }^{54}$ K. Ohta, H. Maekawa, S. Saito, and K. Tominaga, J. Phys. Chem. A 107, 5643 (2003).

${ }^{55}$ K. Ohta, H. Maekawa, and K. Tominaga, J. Phys. Chem. A 108, 1333 (2004).

${ }^{56}$ K. Ohta, H. Maekawa, and K. Tominaga, Chem. Phys. Lett. 386, 32 (2004).

${ }^{57}$ H. Maekawa, K. Ohta, and K. Tominaga, Phys. Chem. Chem. Phys. 6, 4074 (2004).

${ }^{58}$ J. B. Asbury, T. Steinel, and M. Fayer, J. Lumin. 107, 271 (2004).

${ }^{59}$ A. O. Caldeira and A. J. Leggett, Ann. Phys. (N.Y.) 149, 374 (1983).

${ }^{60}$ Y. Tanimura and P. G. Wolynes, Phys. Rev. A 43, 4131 (1991).

${ }^{61}$ Y. Yan, Phys. Rev. A 58, 2721 (1998).

${ }^{62}$ F. Shuang, C. Yang, and Y. Yan, J. Chem. Phys. 114, 3868 (2001).

${ }^{63}$ K. Okumura and Y. Tanimura, Phys. Rev. E 56, 2747 (1997).

${ }^{64}$ V. May and O. Kühn, Charge and Energy Transfer Dynamics in Molecular Systems, 2nd ed. (WILEY-VCH, Weinheim, 2004).

${ }^{65}$ W. H. Miller, J. Chem. Phys. 62, 1899 (1975).

${ }^{66}$ S. Coleman, The Whys of Subnuclear Physics (Plenum, New York, 1979), p. 805 .

${ }^{67}$ G. A. Voth, in New Trends in Kramers' Reaction Rate Theory, Understanding Chemical Reactivity, Vol. 11 (Kluwer, Dordrecht, 1995), p. 216.

${ }^{68}$ Y. Tanimura and R. Kubo, J. Phys. Soc. Jpn. 58, 101 (1989).

${ }^{69}$ R. Kubo, J. Phys. Soc. Jpn. 12, 570 (1957).

${ }^{70}$ R. Kubo, on Stochastic Processes in Chemical Physics, Advances in Chemical Physics, Vol. 15 (Wiley, New York, 1969), p. 101.

${ }^{71}$ Y. Tanimura, H. Takano, and R. Kubo, J. Phys. Soc. Jpn. 55, 4550 (1986).

${ }^{72}$ Y. Tanimura and R. Kubo, J. Phys. Soc. Jpn. 58, 3001 (1989).

${ }^{73}$ Y. Tanimura, T. Suzuki, and R. Kubo, J. Phys. Soc. Jpn. 58, 1850 (1989).

${ }^{74}$ M. Yang and G. R. Fleming, J. Chem. Phys. 110, 2983 (1999).

${ }^{75}$ R. Agarwal, B. S. Prall, A. H. Rizvi, M. Yang, and G. R. Fleming, J. Chem. Phys. 116, 6243 (2002).

${ }^{76}$ T. Kato and Y. Tanimura, Chem. Phys. Lett. 341, 329 (2001).

${ }^{77}$ R. Kubo, M. Toda, and N. Hashitsume, in Statistical Physics II: Nonequilibrium Statistical Mechanics, Springer Series in Solid-State Sciences, Vol. 31, 2nd ed. (Springer, New York, 1991).

${ }^{78}$ H. A. Kramers, Physica (Utrecht) 7, 284 (1940). 
${ }^{79}$ M. Topaler and N. Makri, J. Chem. Phys. 101, 7500 (1994).

${ }^{80}$ T. Hayashi and S. Mukamel, J. Phys. Chem. A 107, 9113 (2003).

${ }^{81}$ R. P. Feynman and F. L. Vernon, Ann. Phys. (N.Y.) 24, 118 (1963).

${ }^{82}$ R. P. Feynman and A. R. Hibbs, Quantum Mechanics and Path Integrals (McGraw-Hill, New York, 1965).

${ }^{83}$ A. O. Caldeira and A. J. Leggett, Physica A 121, 587 (1983).

${ }^{84}$ Y. Tanimura and R. Kubo, J. Phys. Soc. Jpn. 58, 1199 (1989).

${ }^{85}$ D. Chandler, Classical and Quantum Dynamics in Condensed Phase Simulations (World Scientific, Singapore, 1998), p. 3.
${ }^{86}$ D. Chandler, Introduction to Modern Statistical Mechanics (Oxford Unversity Press, New York, 1987).

${ }^{87}$ J. A. Montgomery, Jr., D. Chandler, and B. J. Berne, J. Chem. Phys. 70, 4056 (1978).

${ }^{88}$ P. G. Wolynes, in Lectures in the Sciences of Complexity, Santa Fe Institute Studies in the Science of Complexity, Vol. 1 (Addison-Wesley, Reading, MA, 1989), p. 355.

${ }^{89}$ G. A. Voth, D. Chandler, and W. H. Miller, J. Phys. Chem. 93, 7009 (1989). 